\title{
Interaction of Physical Vacuum with the External Electromagnetic and Gravitational Fields
}

\author{
Boris V. Alexeev \\ Physics Department, Moscow Technological University, Moscow, Russia \\ Email: Boris.Vlad.Alexeev@gmail.com
}

\begin{abstract}
The interaction is established between the Physical Vacuum (PV) description in the frame of non-local physics and the external electromagnetic and gravitational fields.
\end{abstract}

Keywords: Physical vacuum, the Shawyer EM-drive (PV-engines), interaction of physical fields with physical vacuum, Big Bang and the Universe Evolution

\section{Introduction}

Monographs [1 - 4] reveal the following effects of the principal significance:

1. The birth of the universe is convoying of appearance of the repulsion forces. In the existing terminology - we discover the "negative pressure" and "dark energy" in all cases. This fundamental result does not depend on the mechanism of external perturbations. In other words, the anti-gravity in the physical vacuum exists, if there is dissipation of energy or in the absence of dissipation at all.

2. Physical Vacuum (PV) is not a speculative object; it is a reality as "matter" and "fields". In other words, the physical vacuum is "the third" physical reality along with matter and fields. In this case, it is natural to raise the question about the existence of the effect which is similar to the Hubble's effect. As installing the appearance of this effect in the physical vacuum does not contradict the conclusions of non-local physics.

3. In the article [5] we investigated in the frame of non-local physics the connection between from the first glance different effects like Physical Vacuum and PV boxes, clear air turbulence (CAT), the Shawyer EM-drive (PV-engines), Special Theory of Relativity. In the article [6] the wave processes in PV were investigated.

4. We intend to find the solutions of the transport equations defining the evolution the physical vacuum $(\mathrm{PV})$. It means:

A) If the matter is absent, non-local evolution equations have nevertheless non-trivial solutions corresponding evolution of PV which description in time and 3D space on the level of quantum hydrodynamics demands only quantum pressure $p$, the self-consistent force $\mathbf{F}$ (acting on unit of the space volume) and velocity $\mathbf{v}_{\mathbf{0}}$. The system of non local equations is written for the case when the usual matter is absent ( $\rho=0$ ), also radiation, gravitation (as well as other mass forces) and electromagnetic fields are absent. No reason to speak about special or general relativity in this situation, because these theories don't work in the described conditions, (see in particular Items 5 and 6).

B) In all other cases we consider from the position of the nonlocal physics the interaction of Physical Vacuum with the external electromagnetic and gravitational fields taking into account the possible technical applications like EM-engine.

C) Formally speaking the Newtonian gravity propagates with the infinite speed. This conclusion is connected only with the description in the frame of local physics. Usual affirmation - general relativity (GR) reduces to Newtonian gravity in the weak-field, low-velocity limit. In literature you can find criticism of this affirmation because the conservation of angular momentum is implicit in the assumptions on which GR rests. Finite propagation speeds and conservation of angular momentum are incompatible in GR. Therefore, phenomenological GR was forced to claim that gravity is not a force that propagates in any classical sense. But here I do not intend to join to this widely discussed topic using only unified non-local model. The self-consistent description of PV with electromagnetic and gravitational fields originated by PV is the topic of other investigations. 
In the following the nonlocal physics is applied for description of interaction of PV with the external gravitational and electromagnetic fields. All results are obtained from the first principles of physics.

\section{The Basic System of Nonlocal Hydrodynamic Equations}

In monographs [1-4] the evolution of Physical Vacuum (PV) in Planck epoch and wave effects in PV are considered in the frame of nonlocal physics. It should be underlined that the nonlocal transport equations are obtained from the first principles of physics. Here we intend to find the solutions of PV equations for a physical system containing PV and electromagnetic and gravitational fields.

During all investigations we needn't to use the theory Newtonian gravitation or the Maxwell equations in explicit forms for solution of nonlinear non-local evolution equations. In contrast with the local physics this approach in the frame of quantum non-local hydrodynamics leads to the closed mathematical description for the physical system under consideration. If the matter is absent, non-local evolution equations have nevertheless non-trivial solutions corresponding to evolution of PV whose description in time and 3D space on the level of quantum hydrodynamics demands only quantum pressure $p$, the self-consistent force $\mathbf{F}$ (acting on unit of the space volume) and velocity $\mathbf{v}_{0}$.

Moreover, it could be said that we are living in physical vacuum in its form remaining after PV burst (Big Bang) and creation of fields and matter in the following PV evolution. In the frame of nonlocal description it leads to the system of equations [1-4]:

(continuity equation)

$$
\frac{\partial}{\partial t}\left\{\rho-\tau\left[\frac{\partial \rho}{\partial t}+\frac{\partial}{\partial \mathbf{r}} \cdot\left(\rho \mathbf{v}_{0}\right)\right]\right\}+\frac{\partial}{\partial \mathbf{r}} \cdot\left\{\rho \mathbf{v}_{0}-\tau\left[\frac{\partial}{\partial t}\left(\rho \mathbf{v}_{0}\right)+\frac{\partial}{\partial \mathbf{r}} \cdot\left(\rho \mathbf{v}_{0} \mathbf{v}_{0}\right)+\overrightarrow{\mathrm{I}} \cdot \frac{\partial p}{\partial \mathbf{r}}-\mathbf{F}\right]\right\}=0,
$$

(continuity equation, $1 \mathrm{D}$ case; $u$ - velocity in the $x$ - direction)

$$
\frac{\partial}{\partial t}\left\{\rho-\tau\left[\frac{\partial \rho}{\partial t}+\frac{\partial}{\partial x}(\rho u)\right]\right\}+\frac{\partial}{\partial x}\left\{\rho u-\tau\left[\frac{\partial}{\partial t}(\rho u)+\frac{\partial}{\partial x}\left(\rho u^{2}\right)+\frac{\partial p}{\partial x}-F\right]\right\}=0
$$

(momentum equation)

$$
\begin{aligned}
& \frac{\partial}{\partial t}\left\{\rho \mathbf{v}_{0}-\tau\left[\frac{\partial}{\partial t}\left(\rho \mathbf{v}_{0}\right)+\frac{\partial}{\partial \mathbf{r}} \cdot \rho \mathbf{v}_{0} \mathbf{v}_{0}+\frac{\partial p}{\partial \mathbf{r}}-\mathbf{F}\right]\right\}-\mathbf{g}\left[\rho-\tau\left(\frac{\partial \rho}{\partial t}+\frac{\partial}{\partial \mathbf{r}} \cdot\left(\rho \mathbf{v}_{0}\right)\right)\right] \\
& +\frac{\partial}{\partial \mathbf{r}} \cdot\left\{\rho \mathbf{v}_{0} \mathbf{v}_{0}+p \overrightarrow{\mathrm{I}}-\tau\left[\begin{array}{l}
\left.\frac{\partial}{\partial t}\left(\rho \mathbf{v}_{0} \mathbf{v}_{0}+p \overrightarrow{\mathrm{I}}\right)+\frac{\partial}{\partial \mathbf{r}} \cdot \rho\left(\mathbf{v}_{0} \mathbf{v}_{0}\right) \mathbf{v}_{0}+2 \overrightarrow{\mathrm{I}}\left(\frac{\partial}{\partial \mathbf{r}} \cdot\left(p \mathbf{v}_{0}\right)\right)\right] \\
+\frac{\partial}{\partial \mathbf{r}} \cdot\left(\overrightarrow{\mathrm{I}} p \mathbf{v}_{0}\right)-\mathbf{F} \mathbf{v}_{0}-\mathbf{v}_{0} \mathbf{F}
\end{array}\right]\right\}=0
\end{aligned}
$$

(momentum equation, 1D case)

$$
\begin{aligned}
& \frac{\partial}{\partial t}\left\{\rho u-\tau\left[\frac{\partial}{\partial t}(\rho u)+\frac{\partial}{\partial x}\left(\rho u^{2}\right)+\frac{\partial p}{\partial x}-F\right]\right\}-g\left[\rho-\tau\left(\frac{\partial \rho}{\partial t}+\frac{\partial}{\partial x}(\rho u)\right)\right] \\
& +\frac{\partial}{\partial x}\left\{\rho u^{2}+p-\tau\left[\frac{\partial}{\partial t}\left(\rho u^{2}+p\right)+\frac{\partial}{\partial x}\left(\rho u^{3}+3 p u\right)-2 F u\right]\right\}=0,
\end{aligned}
$$

(energy equation)

$$
\begin{aligned}
& \frac{\partial}{\partial t}\left\{\frac{\rho v_{0}^{2}}{2}+\frac{3}{2} p-\tau\left[\frac{\partial}{\partial t}\left(\frac{\rho v_{0}^{2}}{2}+\frac{3}{2} p\right)+\frac{\partial}{\partial \mathbf{r}} \cdot\left(\frac{1}{2} \rho v_{0}^{2} \mathbf{v}_{0}+\frac{5}{2} p \mathbf{v}_{0}\right)-\mathbf{F} \cdot \mathbf{v}_{0}\right]\right\} \\
& +\frac{\partial}{\partial \mathbf{r}} \cdot\left\{\frac{1}{2} \rho v_{0}^{2} \mathbf{v}_{0}+\frac{5}{2} p \mathbf{v}_{0}-\tau\left[\frac{\partial}{\partial t}\left(\frac{1}{2} \rho v_{0}^{2} \mathbf{v}_{0}+\frac{5}{2} p \mathbf{v}_{0}\right)+\frac{\partial}{\partial \mathbf{r}} \cdot\left(\frac{1}{2} \rho v_{0}^{2} \mathbf{v}_{0} \mathbf{v}_{0}+\frac{7}{2} p \mathbf{v}_{0} \mathbf{v}_{0}+\frac{1}{2} p v_{0}^{2} \overrightarrow{\mathrm{I}}\right.\right.\right. \\
& \left.\left.\left.+\frac{5}{2} \frac{p^{2}}{\rho} \overrightarrow{\mathrm{I}}\right)-\mathbf{F} \cdot \mathbf{v}_{0} \mathbf{v}_{0}-p \mathbf{g} \cdot \overrightarrow{\mathrm{I}}-\frac{1}{2} v_{0}^{2} \mathbf{F}-\frac{3}{2} \mathbf{g} p\right]\right\}-\left\{\mathbf{F} \cdot \mathbf{v}_{0}-\tau\left[\mathbf{g} \cdot\left(\frac{\partial}{\partial t}\left(\rho \mathbf{v}_{0}\right)+\frac{\partial}{\partial \mathbf{r}} \cdot \rho \mathbf{v}_{0} \mathbf{v}_{0}+\frac{\partial}{\partial \mathbf{r}} \cdot p \vec{I}-\mathbf{F}\right)\right]\right\}=0,
\end{aligned}
$$

(energy equation, 1D case) 


$$
\begin{aligned}
& \frac{\partial}{\partial t}\left\{\rho u^{2}+3 p-\tau\left[\frac{\partial}{\partial t}\left(\rho u^{2}+3 p\right)+\frac{\partial}{\partial x}\left(\rho u^{3}+5 p u\right)-2 F u\right]\right\} \\
& +\frac{\partial}{\partial x}\left\{\rho u^{3}+5 p u-\tau\left[\frac{\partial}{\partial t}\left(\rho u^{3}+5 p u\right)+\frac{\partial}{\partial x}\left(\rho u^{4}+8 p u^{2}\right)-2 F u^{2}-u^{2} F\right]\right\}+5 \frac{\partial}{\partial x}\left\{\tau\left(\frac{p}{\rho} F-\frac{\partial}{\partial x} \frac{p^{2}}{\rho}\right)\right\} \\
& -2\left\{F u-\tau g\left(\frac{\partial}{\partial t}(\rho u)+\frac{\partial}{\partial x}\left(\rho u^{2}\right)+\frac{\partial p}{\partial x}-F\right)\right\}=0,
\end{aligned}
$$

Here $p$ is pressure of PV, $u$ is velocity of PV expanding, and $F$ is the self consistent force acting in $\mathrm{PV}, \tau$ is nonlocal parameter. Nonlinear evolution equations (2.1) - (2.6) contain the forces $\mathbf{F}, \mathbf{g}$ acting on space and masses including cross-term (see for example the last line in equation (2.6)). The relation $\mathbf{F}=\rho \mathbf{g}$ comes into being only after the mass appearance as result of the PV explosion.

We intend to find the solutions of the transport equations defining the evolution of the physical vacuum $(\mathrm{PV})$. It means:

1. The system of non-local equations should be written for the case when the usual matter is absent $(\rho=0)$, but gravitation and electromagnetic fields are not absent.

2. Physical Vacuum is "one species" system.

Let us apply generalized quantum hydrodynamic equations for investigation of the evolution PV using (for better understanding) stationary 1D Cartesian description, $F_{x}=F$ is the force acting in the positive $x$ - direction. We have in this limit case $\rho \rightarrow 0$ (see also [1-6]) from the continuity equation

$$
\frac{\partial}{\partial x}\left\{\tau\left[\frac{\partial p}{\partial x}-F\right]\right\}=0
$$

from the momentum equation

$$
\begin{aligned}
& \left(\frac{\partial p}{\partial x}-F\right)-2 \tau\left(\frac{\partial p}{\partial x}-F\right) \frac{\partial u}{\partial x}- \\
& -\frac{\partial}{\partial x}\left\{\tau\left[3 p \frac{\partial u}{\partial x}+u \frac{\partial p}{\partial x}\right]\right\}=0 .
\end{aligned}
$$

Transform now the energy equation (1D stationary case) (2.6) using some intermediate transformations

$$
\begin{aligned}
& \frac{\partial}{\partial x}\left\{5 p u-\tau\left[\frac{\partial}{\partial x}\left(8 p u^{2}\right)-3 F u^{2}\right]\right\}+5 \frac{\partial}{\partial x}\left\{\tau\left(p g-\frac{\partial}{\partial x} \frac{p^{2}}{\rho}\right)\right\} \\
& -2\left\{F u-2 \tau F u \frac{\partial u}{\partial x}-\tau g\left(\frac{\partial p}{\partial x}-F\right)\right\}=0,
\end{aligned}
$$

or

$$
\begin{aligned}
& 5 u \frac{\partial p}{\partial x}+5 p \frac{\partial u}{\partial x}-2 F u-\frac{\partial}{\partial x}\left\{\tau\left[8 \frac{\partial}{\partial x}\left(p u^{2}\right)-3 F u^{2}-5 p g\right]\right\} \\
& +4 \tau F u \frac{\partial u}{\partial x}+2 \tau g\left(\frac{\partial p}{\partial x}-F\right)-5 \frac{\partial}{\partial x}\left\{\tau \frac{\partial}{\partial x} \frac{p^{2}}{\rho}\right\}=0 .
\end{aligned}
$$

In the energy equation (2.10) the term $A^{\text {pert }}$ defines the possible perturbation of physical vacuum as a result of the perturbation appearance like Higgs boson.

$$
A^{\text {pert }}=-5 \frac{\partial}{\partial x}\left\{\tau \frac{\partial}{\partial x} \frac{p^{2}}{\rho}\right\}
$$

If gradient of the initial energy perturbation is small

$$
A^{\text {pert }} \cong 0
$$

We write down the system of equations (2.7)-(2.9) and (2.11) using the simplest solution (2.7)

$$
\frac{\partial p}{\partial x}=F
$$


As we see in the simplest case the PV pressure $p$ can be associated with potential of energy. We find from (2.10)

$$
\begin{aligned}
& 5 u \frac{\partial p}{\partial x}+5 p \frac{\partial u}{\partial x}-2 F u-\frac{\partial}{\partial x}\left\{\tau\left[16 u p \frac{\partial u}{\partial x}+8 u^{2} \frac{\partial p}{\partial x}-3 u^{2} F-5 p g\right]\right\} \\
& +4 \tau F u \frac{\partial u}{\partial x}=0 .
\end{aligned}
$$

or

$$
\begin{aligned}
& 3 u \frac{\partial p}{\partial x}+5 p \frac{\partial u}{\partial x}-\frac{\partial}{\partial x}\left\{\tau\left[16 u p \frac{\partial u}{\partial x}+5 u^{2} \frac{\partial p}{\partial x}-5 p g\right]\right\} \\
& +4 \tau u \frac{\partial p}{\partial x} \frac{\partial u}{\partial x}=0 .
\end{aligned}
$$

The next simplifying proposition is $\tau=$ const and we simplify equation (2.15):

$$
\begin{aligned}
& 3 u \frac{\partial p}{\partial x}+5 p \frac{\partial u}{\partial x}-\tau \frac{\partial}{\partial x}\left[16 u p \frac{\partial u}{\partial x}+5 u^{2} \frac{\partial p}{\partial x}-5 p g\right] \\
& +4 \tau u \frac{\partial p}{\partial x} \frac{\partial u}{\partial x}=0 .
\end{aligned}
$$

Using (2.13) we obtain the simplified momentum equation

and the energy equation

$$
3 p \frac{\partial u}{\partial x}+u \frac{\partial p}{\partial x}=0
$$

$$
\begin{aligned}
& 4 p \frac{\partial u}{\partial x}+\tau \frac{\partial}{\partial x}\left[16 u p \frac{\partial u}{\partial x}+5 u^{2} \frac{\partial p}{\partial x}-5 p g\right]- \\
& -4 u \tau \frac{\partial p}{\partial x} \frac{\partial u}{\partial x}=0,
\end{aligned}
$$

Write down equations $(2.13),(2.17),(2.18)$ in the dimensionless forms using the scales

$$
\left[x_{0}\right],\left[u_{0}\right],\left[p_{0}\right],[F]=\frac{p_{0}}{x_{0}},\left[t_{0}\right]=\frac{x_{0}}{u_{0}}, \rho_{0}, x_{0}=u_{0} t_{0}, p_{0}=\rho_{0} u_{0}^{2} .
$$

and tilde for the dimensionless values; we reach SYSTEM 1:

$$
\begin{gathered}
\frac{\partial \tilde{p}}{\partial \tilde{x}}=\tilde{F} \\
3 \tilde{p} \frac{\partial \tilde{u}}{\partial \tilde{x}}+\tilde{u} \frac{\partial \tilde{p}}{\partial \tilde{x}}=0 \\
4 \tilde{p} \frac{\partial \tilde{u}}{\partial \tilde{x}}+\tau \frac{\partial}{\partial \tilde{x}}\left[16 \tilde{u} \tilde{p} \frac{\partial \tilde{u}}{\partial \tilde{x}}+5 \tilde{u}^{2} \frac{\partial \tilde{p}}{\partial \tilde{x}}-5 \tilde{p} \tilde{g}\right]-4 \tilde{u} \tilde{\tau} \frac{\partial \tilde{p}}{\partial \tilde{x}} \frac{\partial \tilde{u}}{\partial \tilde{x}}=0,
\end{gathered}
$$

Obviously in the limit case of local description $(\tau=0)$ SYSTEM 1 leads to trivial solutions.

Let us consider now the process of excitation of physical vacuum by radiation. It is known that the first experiments demonstrating the direct light pressure on a surface (including gases) were realized by P.N. Lebedev [7]. Then when light impinges on the surface of a liquid, part of the light is reflected (with the reflection coefficient $\chi$ ) and the remaining fraction is transmitted. The new experiments show for the first time that the liquid surface bends inward, meaning that the light is pushing on the fluid in agreement with the Abraham momentum $p_{A}$ of light. The corresponding equation for the photon momentum in a dielectric with refractive index $n$ is:

$$
p_{A}=\frac{h v}{n c}
$$

where $h$ is the Plank constant, $v$ is the frequency of the light and $c$ is the speed of light in vacuum. Light pressure can be found by the formula 


$$
p_{r}=\frac{\Phi_{r}}{c}(\chi+1)
$$

where $\Phi_{r}$ is the density of radiation energy flux falling on a surface, for the mirror surface $\chi=1$. For the state close to thermodynamic equilibrium we have

$$
p_{r}=\frac{U}{3}
$$

where $U$ is the energy density.

Therefore we can introduce in system of equations (2.19) - (2.21) an external dimensionless pressure $\tilde{A}^{e x}$. Generally speaking $\tilde{A}^{e x}$ is a function of coordinates and time and should be calculated independently with the help of the Maxwell equations.

As a result (if the mass perturbation can be omitted) we have the SYSTEM 2:

$$
\begin{gathered}
\frac{\partial}{\partial \tilde{x}}\left(\tilde{p}+\tilde{A}^{e x}\right)=\tilde{F} \\
3\left(\tilde{p}+\tilde{A}^{e x}\right) \frac{\partial \tilde{u}}{\partial \tilde{x}}+\tilde{u} \frac{\partial}{\partial \tilde{x}}\left(\tilde{p}+\tilde{A}^{e x}\right)=0, \\
4\left(\tilde{p}+\tilde{A}^{e x}\right) \frac{\partial \tilde{u}}{\partial \tilde{x}}+ \\
\tau \frac{\partial}{\partial \tilde{x}}\left[16 \tilde{u}\left(\tilde{p}+\tilde{A}^{e x}\right) \frac{\partial \tilde{u}}{\partial \tilde{x}}+5 \tilde{u}^{2} \frac{\partial}{\partial \tilde{x}}\left(\tilde{p}+\tilde{A}^{e x}\right)-5\left(\tilde{p}+\tilde{A}^{e x}\right) \tilde{g}\right]- \\
4 \tilde{u} \tilde{\tau} \frac{\partial \tilde{u}}{\partial \tilde{x}} \frac{\partial}{\partial \tilde{x}}\left(\tilde{p}+\tilde{A}^{e x}\right)=0 .
\end{gathered}
$$

\section{Results of Mathematical Modeling for SYSTEM 1}

Now we are ready to display the results of the mathematical modeling realized with the help of Maple (the versions Maple 9 or more can be used). The SYSTEM 1 has the great possibilities of mathematical modeling as result of changing of four Cauchy conditions and parameters $\tilde{\tau} \tilde{g}_{x}$ and $\tilde{A}^{\text {ex }}$ describing the character features of physical system.

Maple program contains Maple's notations - for example the expression $D(\tilde{u})(0)=0$ means in the usual notations $(\partial \tilde{u} / \partial \tilde{x})(0)=0$, independent variable $t$ responds to $\tilde{x}$. The following Maple notations on figures are used: u- velocity $\tilde{u}, \mathrm{p}$ - pressure $\tilde{p}$, and $\mathrm{f}$ - the self consistent force $\tilde{F}, \mathrm{~A}-\tilde{A}^{e x}, \mathrm{G}-\tilde{g}_{x}$, $\mathrm{T}-\tilde{\tau}$. Explanations placed under all following figures. The results of the calculations are presented in figures 3.1 - 3.19. The information required is contained in the figures and in figure captions. We use for all calculations reflected on figures 3.1 - 3.11 the Cauchy conditions

$$
\tilde{u}(0)=1, \tilde{p}(0)=1, D(\tilde{u})(0)=1, D(\tilde{p})(0)=1,
$$

which of course can be changed; parameters $\tilde{A}$ and $\tilde{g}_{x}$ vary widely. As a rule we use the following lines: $\tilde{u}$ - solid line, $\tilde{p}$ - dashed line, $\partial \tilde{p} / \partial \tilde{x}$ - dotted line.

Remarks:

1. If $D(\tilde{u})(0)=0, D(\tilde{p})(0)=0$ and $\tilde{A}^{e x}=$ const, we have only trivial solutions $\tilde{u}=$ const, $\tilde{p}=$ const. Conditions $D(\tilde{u})(0) \neq 0, D(\tilde{p})(0) \neq 0$ can be considered as a "ruffle" in PV and deliver the appearance of non trivial solutions even if the mass perturbation $A^{\text {pert }}=0$.

2. The figures 3.1 - 3.7 are constructed for the case when the external electromagnetic field is absent $\left(\tilde{A}^{e x}=0\right)$. The left and right boundaries of the solution existence are indicated as lim1 and lim2 correspondingly. Captions like lim1 reflect the domain of the solution existence. 


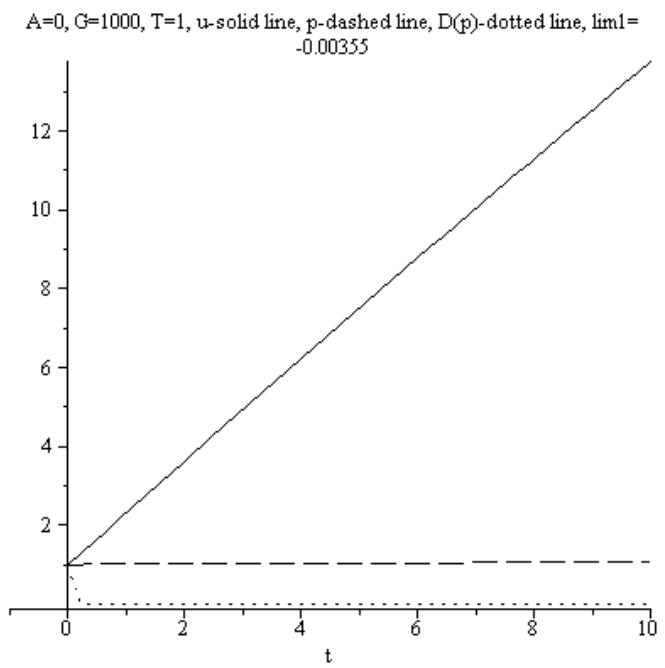

Figure 3.1. Evolution of $\tilde{u}(\tilde{x}), \tilde{p}(\tilde{x}), \partial \tilde{p}(\tilde{x}) / \partial \tilde{x} ; \tilde{A}^{e x}=0, \tilde{\tau}=1, \tilde{g}_{x}=1000$

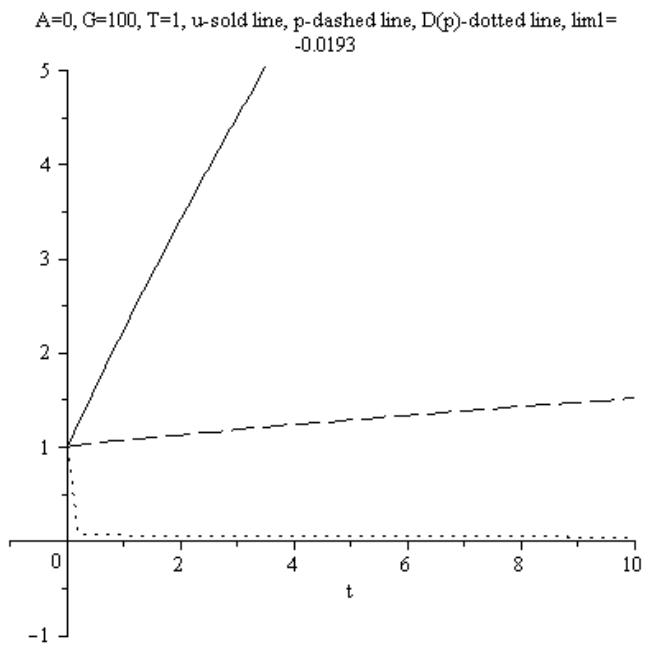

Figure 3.2. Evolution of $\tilde{u}(\tilde{x}), \tilde{p}(\tilde{x}), \partial \tilde{p}(\tilde{x}) / \partial \tilde{x} ; \tilde{A}^{e x}=0, \tilde{\tau}=1, \tilde{g}_{x}=100$

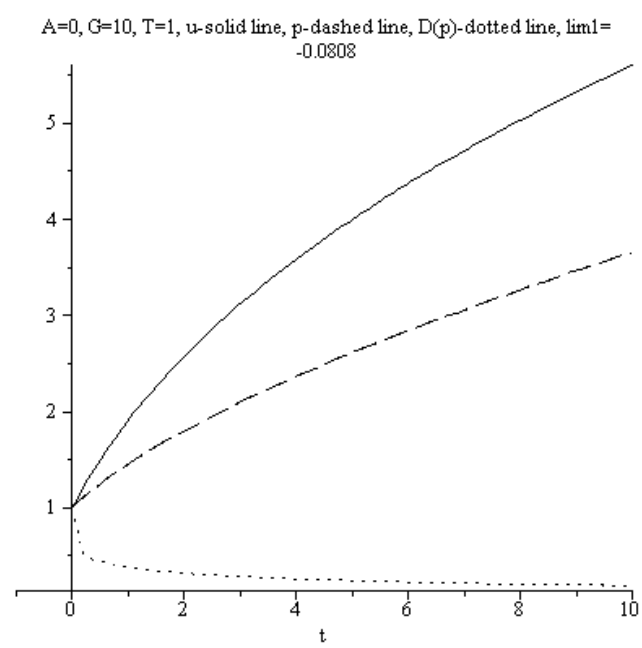

Figure 3.3. Evolution of $\tilde{u}(\tilde{x}), \tilde{p}(\tilde{x}), \partial \tilde{p}(\tilde{x}) / \partial \tilde{x} ; \tilde{A}^{e x}=0, \tilde{\tau}=1 \quad \tilde{g}_{x}=10$ 


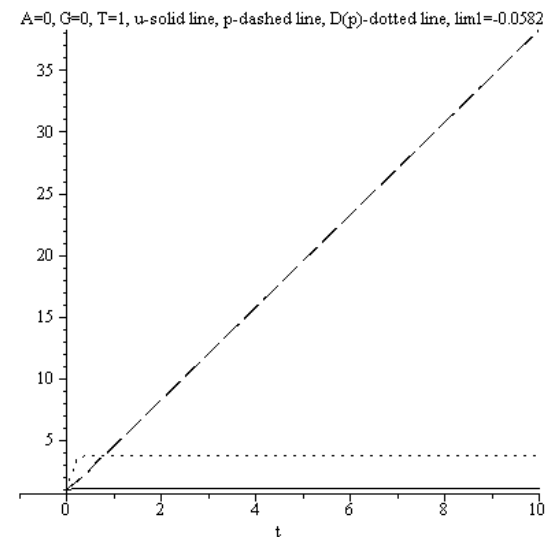

Figure 3.4. Evolution of $\tilde{u}(\tilde{x}), \tilde{p}(\tilde{x}), \partial \tilde{p}(\tilde{x}) / \partial \tilde{x} ; \tilde{A}^{e x}=0, \tilde{\tau}=1, \tilde{g}_{x}=0$

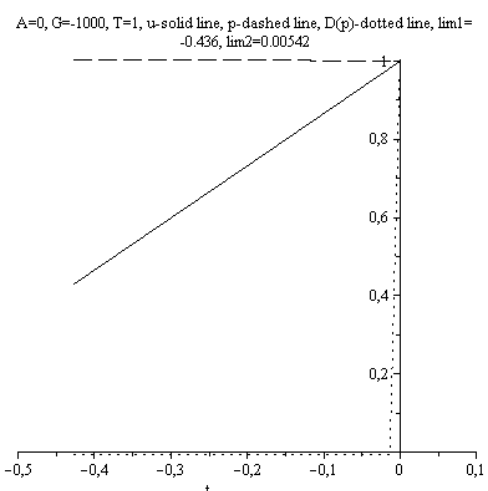

Figure 3.5. Evolution of $\tilde{u}(\tilde{x}), \tilde{p}(\tilde{x}), \partial \tilde{p}(\tilde{x}) / \partial \tilde{x} ; \tilde{A}^{e x}=0, \tilde{\tau}=1, \tilde{g}_{x}=-1000$

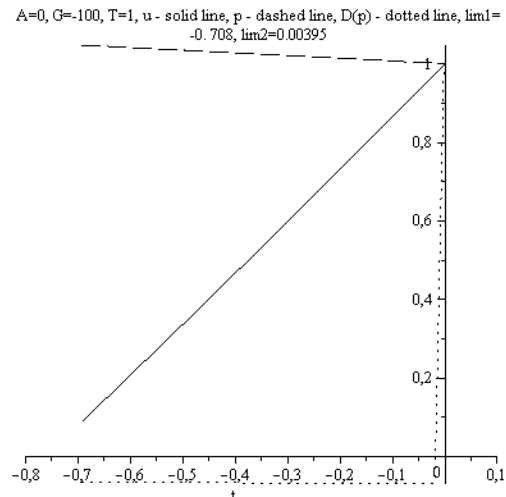

Figure 3.6. Evolution of $\tilde{u}(\tilde{x}), \tilde{p}(\tilde{x}), \partial \tilde{p}(\tilde{x}) / \partial \tilde{x} ; \tilde{A}^{e x}=0, \tilde{\tau}=1, \tilde{g}_{x}=-100$ $\mathrm{A}=0, \mathrm{G}=-10, \mathrm{~T}=1, \mathrm{w}-\mathrm{solid}$ line, $\mathrm{p}-\mathrm{dashed}$ line, $\mathrm{D}(\mathrm{p})$-dotted line. $\operatorname{lim1} 1=$

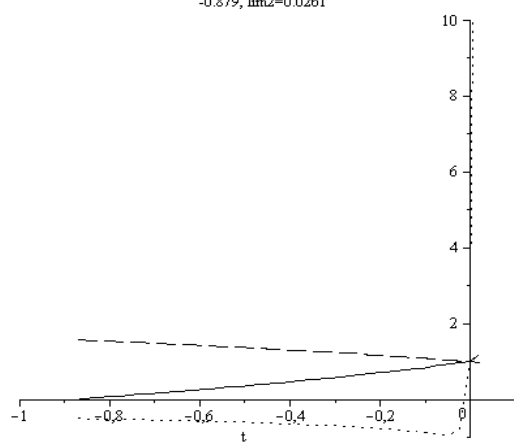

Figure 3.7. Evolution of $\tilde{u}(\tilde{x}), \tilde{p}(\tilde{x}), \partial \tilde{p}(\tilde{x}) / \partial \tilde{x} ; \tilde{A}^{e x}=0, \tilde{\tau}=1, \tilde{g}_{x}=-10$ 
The following figures $3.8-3.10$ are constructed for the same Cauchy conditions $\tilde{u}(0)=1, \tilde{p}(0)=1$, $D(\tilde{u})(0)=1, D(\tilde{p})(0)=1$ but for the parameter $\tilde{A}^{e x}=1$ reflecting the appearance the external constant pressure radiation.

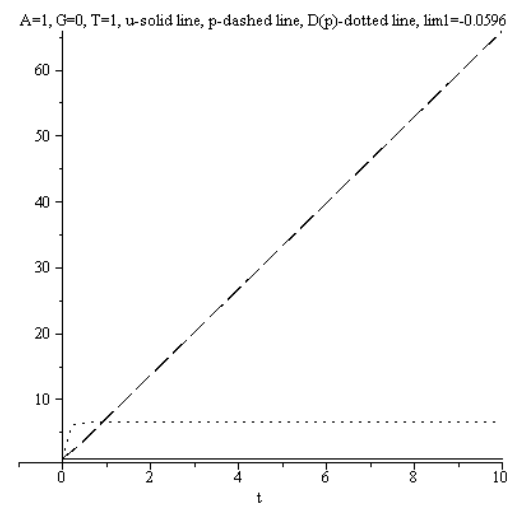

Figure 3.8. Evolution of $\tilde{u}(\tilde{x}), \tilde{p}(\tilde{x}), \partial \tilde{p}(\tilde{x}) / \partial \tilde{x} ; \tilde{A}^{e x}=1, \tilde{\tau}=1, \tilde{g}_{x}=0$

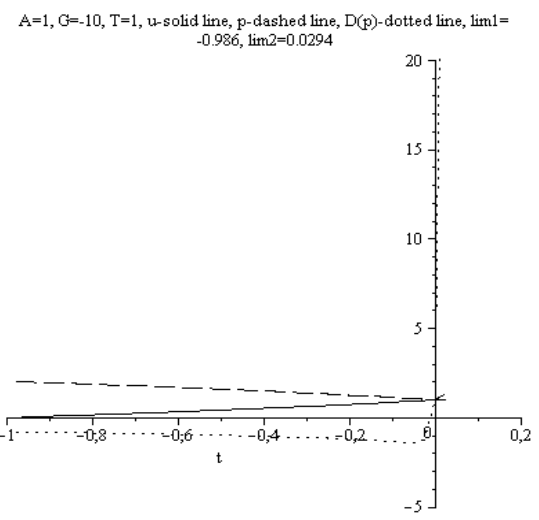

Figure 3.9. Evolution of $\tilde{u}(\tilde{x}), \tilde{p}(\tilde{x}), \partial \tilde{p}(\tilde{x}) / \partial \tilde{x} ; \tilde{A}^{e x}=1, \tilde{\tau}=1, \tilde{g}_{x}=-10$

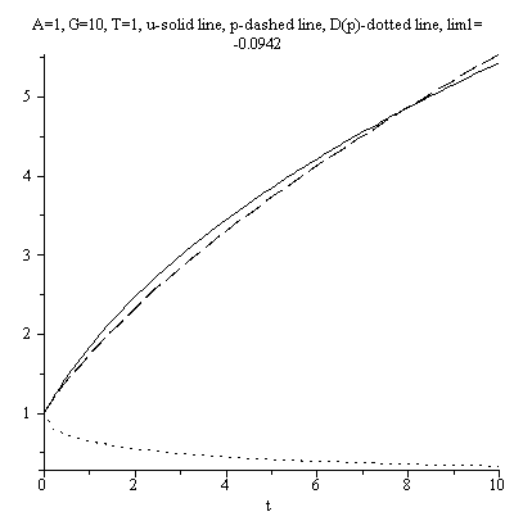

Figure 3.10. Evolution of $\tilde{u}(\tilde{x}), \tilde{p}(\tilde{x}), \partial \tilde{p}(\tilde{x}) / \partial \tilde{x} ; \tilde{A}^{e x}=1, \tilde{\tau}=1, \tilde{g}_{x}=10$

The following figure 3.11 is constructed for the same Cauchy conditions $\tilde{u}(0)=1, \tilde{p}(0)=1$, $D(\tilde{u})(0)=1, D(\tilde{p})(0)=1$ but for the parameter $\tilde{A}^{e x}=100$ reflecting the influence of the more strong external radiation. 


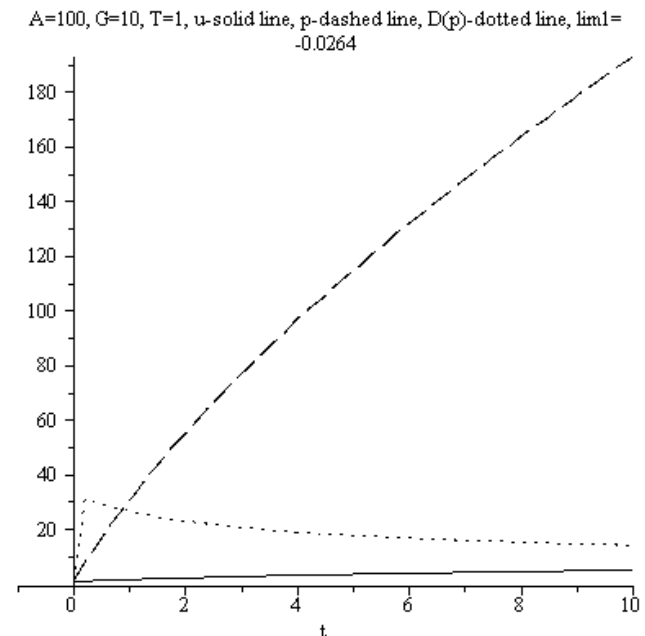

Figure 3.11. Evolution of $\tilde{u}(\tilde{x}), \tilde{p}(\tilde{x}), \partial \tilde{p}(\tilde{x}) / \partial \tilde{x} ; \tilde{A}^{e x}=100, \tilde{\tau}=1, \tilde{g}_{x}=10$

The figures $3.12-3.17$ show the result of modeling for different constant $\tilde{A}$ with the Cauchy conditions: $p(0)=1, u(0)=1, D(p)(0)=-1, \quad D(u)(0)=-1$.

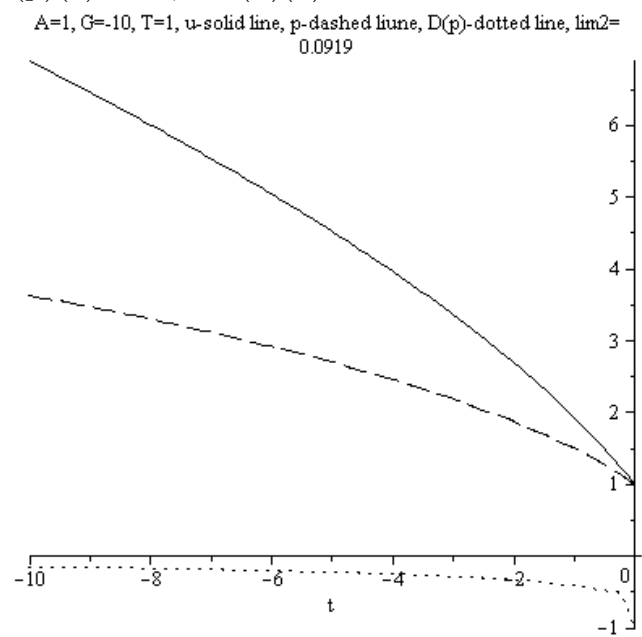

Figure 3.12. Evolution of $\tilde{u}(\tilde{x}), \tilde{p}(\tilde{x}), \partial \tilde{p}(\tilde{x}) / \partial \tilde{x} ; \tilde{A}=1, \tilde{\tau}=1, \tilde{g}_{x}=-10, \tilde{x}$ interval $(-10,1)$

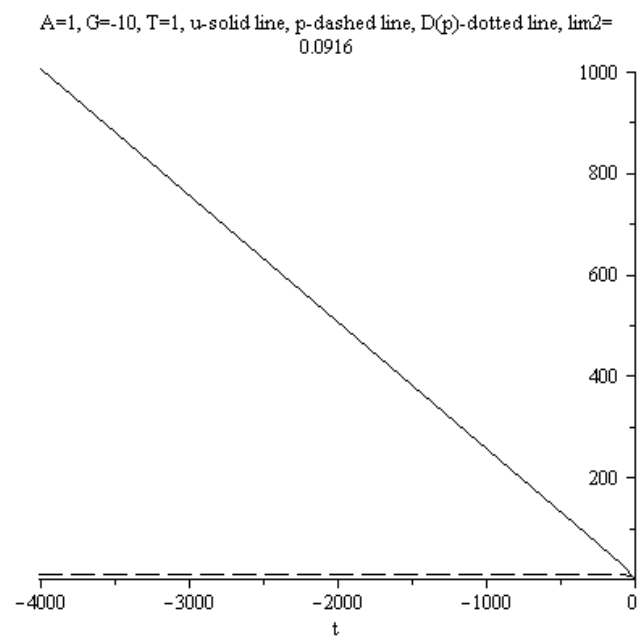

Figure 3.13. Evolution of $\tilde{u}(\tilde{x}), \tilde{p}(\tilde{x}), \partial \tilde{p}(\tilde{x}) / \partial \tilde{x} ; \tilde{A}=1, \tilde{\tau}=1, \tilde{g}_{x}=-10, \tilde{x}$ interval $(-4000,1)$ 


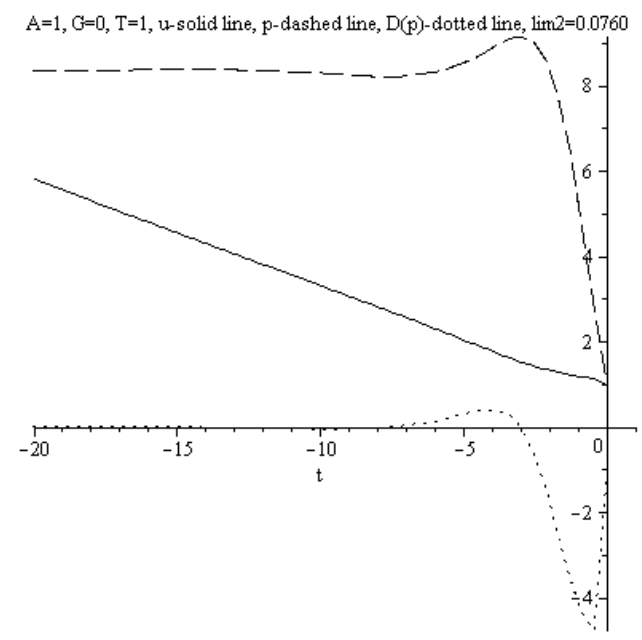

Figure 3.14. Evolution of $\tilde{u}(\tilde{x}), \tilde{p}(\tilde{x}), \partial \tilde{p}(\tilde{x}) / \partial \tilde{x} ; \tilde{A}=1, \tilde{\tau}=1, \tilde{g}_{x}=0, \tilde{x}$ interval $(-20,1)$.

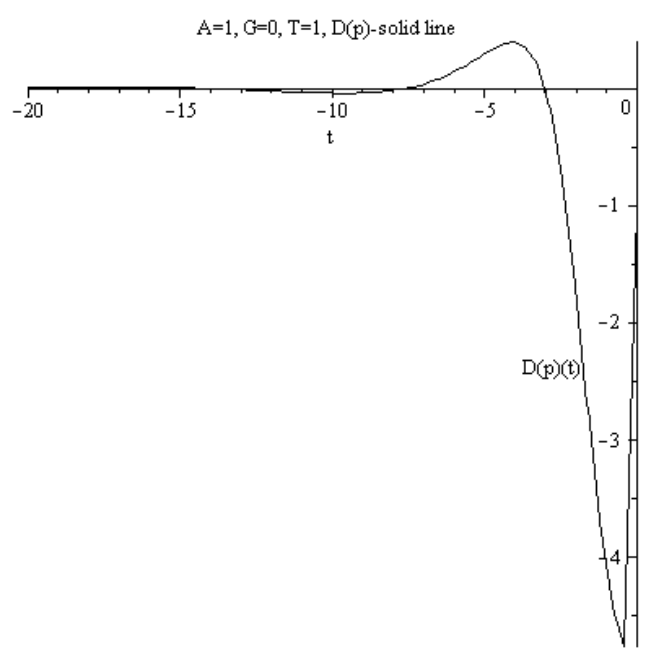

Figure 3.15. Evolution of $\partial \tilde{p}(\tilde{x}) / \partial \tilde{x}=\tilde{F}(\tilde{x}) ; \tilde{A}=1, \tilde{\tau}=1, \tilde{g}_{x}=0$

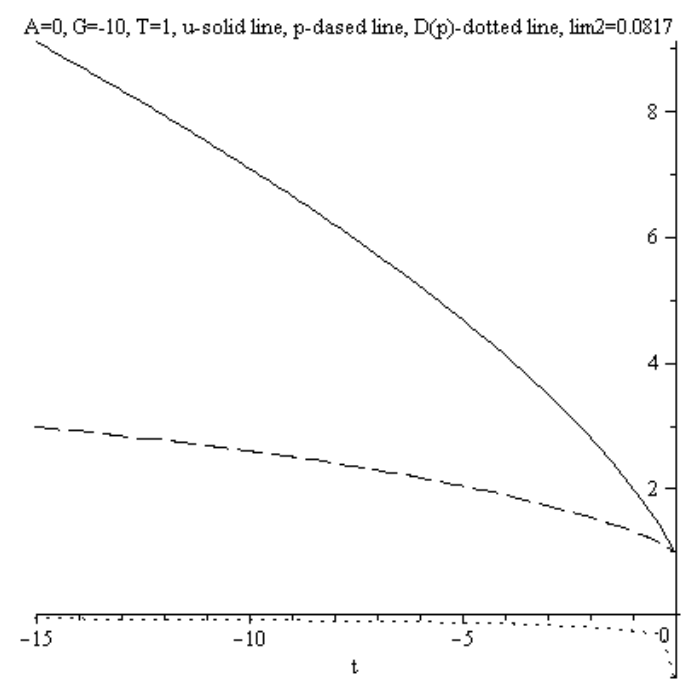

Figure 3.16. Evolution of $\tilde{u}(\tilde{x}), \tilde{p}(\tilde{x}), \partial \tilde{p}(\tilde{x}) / \partial \tilde{x} ; \tilde{A}=0, \tilde{\tau}=1, \tilde{g}_{x}=-10$ 


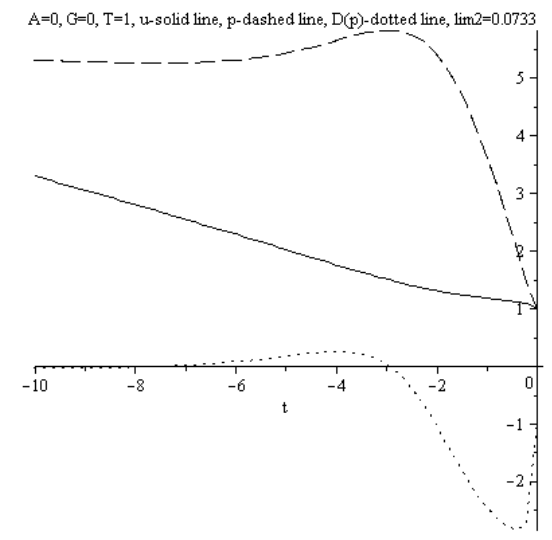

Figure 3.17. Evolution of $\tilde{u}(\tilde{x}), \tilde{p}(\tilde{x}), \partial \tilde{p}(\tilde{x}) / \partial \tilde{x} ; \tilde{A}=0, \tilde{\tau}=1, \tilde{g}_{x}=0$

From the calculations reflected on figures 3.15 and 3.17 follow that the space evolution of the force $\tilde{F}(\tilde{x})$ can have the character of the damped oscillations.

The figures 3.18, 3.19 show the result of modeling for $\tilde{A}=1$ with the Cauchy conditions: $p(0)=1, u(0)=1, D(p)(0)=-1, \quad D(u)(0)=-1$ but with the opposite direction of the gravity force and the initial gradients of pressure and velocity. In this case the corresponding solutions occupy the finite space interval.

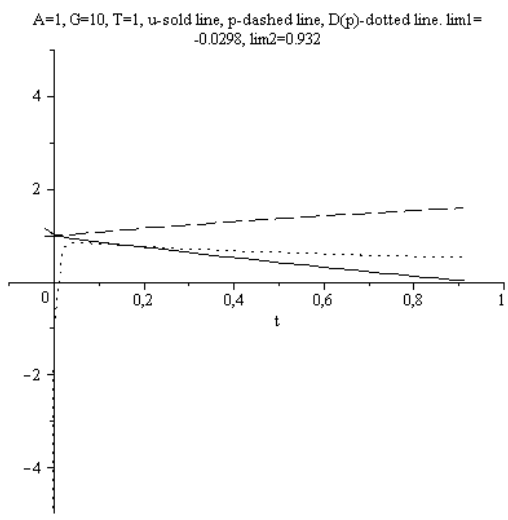

Figure 3.18. Evolution of $\tilde{u}(\tilde{x}), \tilde{p}(\tilde{x}), \partial \tilde{p}(\tilde{x}) / \partial \tilde{x} ; \tilde{A}=1, \tilde{\tau}=1, \tilde{g}_{x}=10$

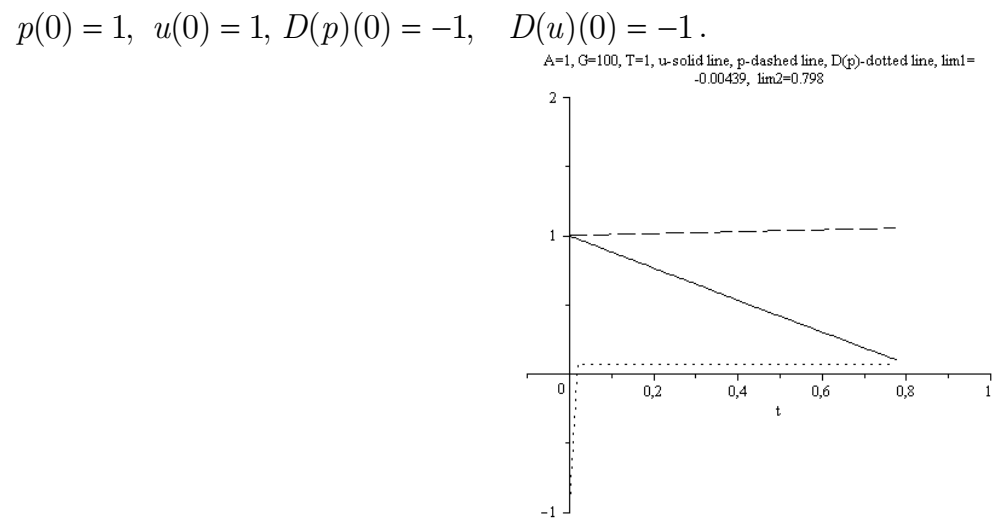

Figure 3.19. Evolution of $\tilde{u}(\tilde{x}), \tilde{p}(\tilde{x}), \partial \tilde{p}(\tilde{x}) / \partial \tilde{x} ; \tilde{A}=1, \tilde{\tau}=1, \tilde{g}_{x}=100$

$p(0)=1, u(0)=1, D(p)(0)=-1, \quad D(u)(0)=-1$. 


\section{To the Theory of PV-Engines}

Let us discuss now from the position of the developed theory the situation with the so called "EM Drive". This (hypothetical) engine was invented by British scientist Roger Shawyer in 1999. The principal scheme of this EM Drive can be shown as follows (Fig. 4.1):

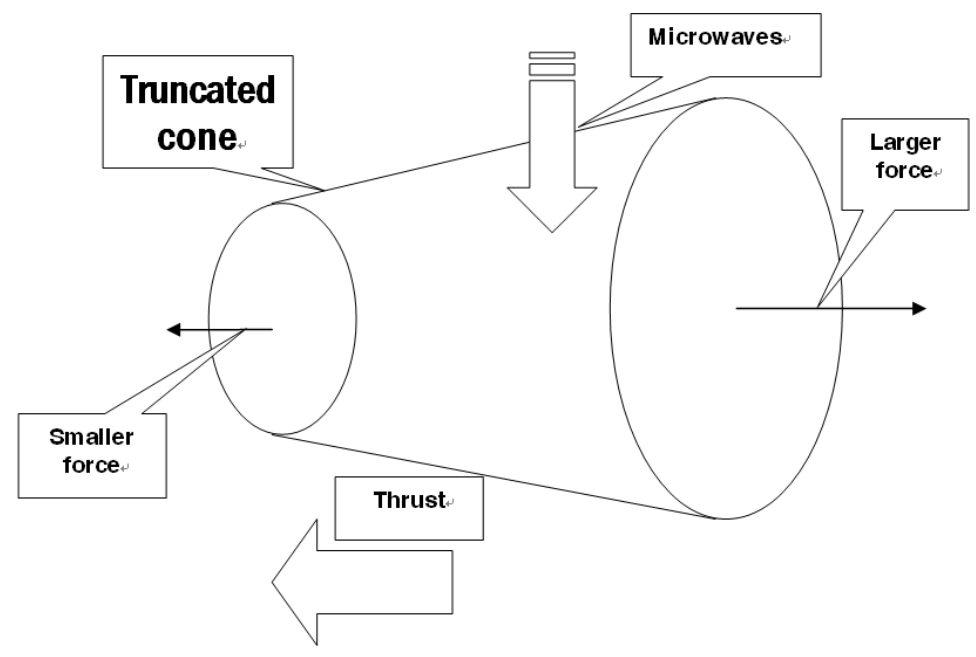

Figure 4.1. Principal scheme of EM Drive.

Shawyer's testing was done on a torsion balance using air bearings ([8], see also $[9,10])$. He observed rotation of the complete apparatus with all electronics and power supplies on-board.

The typical parameters of following White's experiments [11-13] are as follows. The RF resonance test article is a copper frustum with an inner diameter of $27.9 \mathrm{~cm}$ on the big end, an inner diameter of 15.9 $\mathrm{cm}$ on the small end, and an axial length of $22.9 \mathrm{~cm}$. The vacuum test campaign consisted of a forward thrust phase and reverse thrust phase at less than $8 \times 10^{-6}$ torr vacuum with power scans at 40,60, and $80 \mathrm{~W}$. The test campaign included a null thrust test effort to identify any mundane sources of impulsive thrust; however, none were identified. Thrust data from forward, reverse, and null suggested that the system was consistently performing with a thrust-to-power ratio of $1.2 \pm 0.1 \mathrm{mNkW}$.

The usual comment for the thrust appearance in this construction sounds as follows. The EM Drive uses electromagnetic waves as "fuel", creating thrust by bouncing microwave photons back and forth inside a cone-shaped closed metal cavity. In other words, electricity converts into microwaves within the cavity that push against the inside of the device, causing the thruster to accelerate in the opposite direction.

Obviously this explanation has no attitude to reality. The nozzle of this "jet engine" is closed by a round plate. It means that the formulated explanation leads to the contradiction with the Newton's Third Law, which states, "To each action there's an equal and opposite reaction," and many physicists say the EM Drive categorically violates that law. From the position of classical mechanics this corresponds to the attempt of Baron Münchhausen to pull itself out of the swamp by his own hair. In order for a thruster to gain momentum in a certain direction, it has to expel some kind of propellant or exhaust in the opposite direction. But the EM Drive knows nothing about the law of conservation of momentum, which Newton derived from his Third Law.

Since its invention, the EM drive was tested many times and reveals "anomalous thrust signals". Putting it mildly, we can say - if EM Drive indeed produces thrust we should find the corresponding explanation for this effect.

In this case I should define my position in connection with the mentioned problem:

1. Appearance of thrust in the systems like EM Drive does not contradict the conclusions following from nonlocal physics.

2. The emergence of the thrust due to the interaction of radiation with physical vacuum.

3. It is impossible to provide an explanation of the effect using methods of local physics.

4. Then no reason to discuss other theoretical models originated by local physics. 
5. We do not intend to go into details of the experiment organization including the possible experimental errors. For us the only interesting thing is the correspondence between theoretical and experimental data in basic experiments.

From this point of view the experimental results for emdrive reflected in the Internet site http://www.masinaelectrica.com/emdrive-independent-test/ are interesting for us. These experiments have the very important feature - emdrive was placed in the vertical position. As a result it was revealed the direct influence of gravitation on the emdrive thrust. The experimenter had rather modest equipment and no surprise that he did not observe the horizontal thrust which leads to $\sim \mathrm{mN} / \mathrm{kW}$. But he discovered the more thrust for the vertical emdrive position depending on the orientation emdrive in the vertical plane $(\sim 0.2-1.4$ grammas).

Compare now the figures 3.1-3.3 and 3.5-3.7 with results reflected on figure 3.4 with the absence of the gravitational influence (the usual horizontal balance). We see the strong influence of gravitational field on the PV behavior. The appearance of radiation does not change this conclusion of the principal significance (see for example figures 3.4, and 3.8-3.11)

The physical vacuum is moved in the direction of action of gravity (compare figures 3.1- 3.3 and 3.53.7). It should be noticed the strong influence of Cauchy conditions (PV perturbations) on the PV space evolution.

The calculations presented on figures 3.1-3.11 give a general idea of the physical system filled by radiation, PV and gravitation. But radiation occupies only a part of the considered system diminishing with the grows of the distance $\tilde{x}$ Let us reflect this fact introducing the approximation

$$
\tilde{A}^{e x}=\frac{\tilde{B}}{1+\tilde{x}^{n}}
$$

The following figures 4.2-4.6 show the result of modeling for approximation (4.1) for different $\tilde{B}$ and $n$ with the Cauchy conditions: $p(0)=1, u(0)=1, D(p)(0)=1, D(u)(0)=1$.

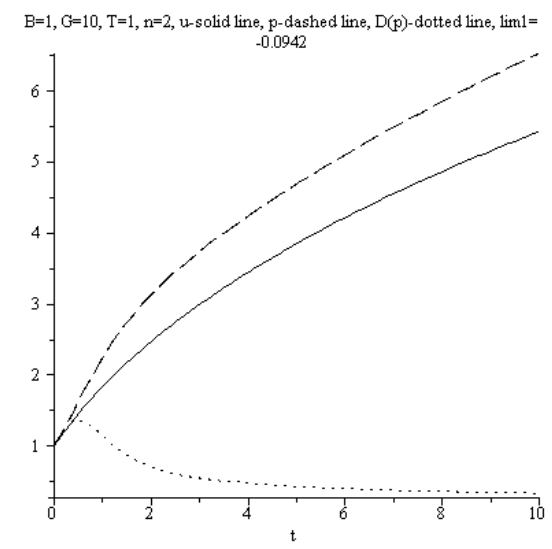

Figure 4.2. Evolution of $\tilde{u}(\tilde{x}), \tilde{p}(\tilde{x}), \partial \tilde{p}(\tilde{x}) / \partial \tilde{x} ; \tilde{B}=1, \tilde{\tau}=1, \tilde{g}_{x}=10, n=2$

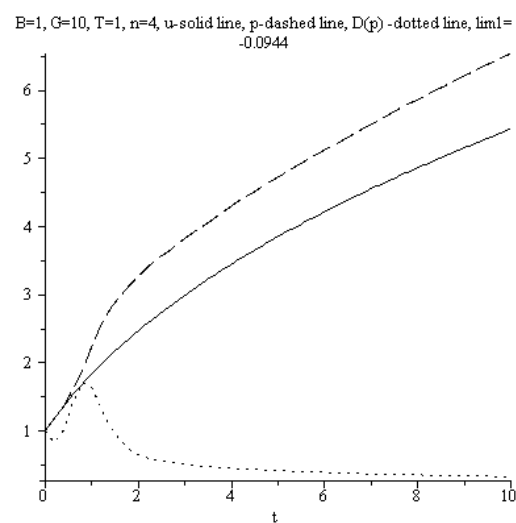

Figure 4.3. Evolution of $\tilde{u}(\tilde{x}), \tilde{p}(\tilde{x}), \partial \tilde{p}(\tilde{x}) / \partial \tilde{x} ; \tilde{B}=1, \tilde{\tau}=1, \tilde{g}_{x}=10, n=4$ 


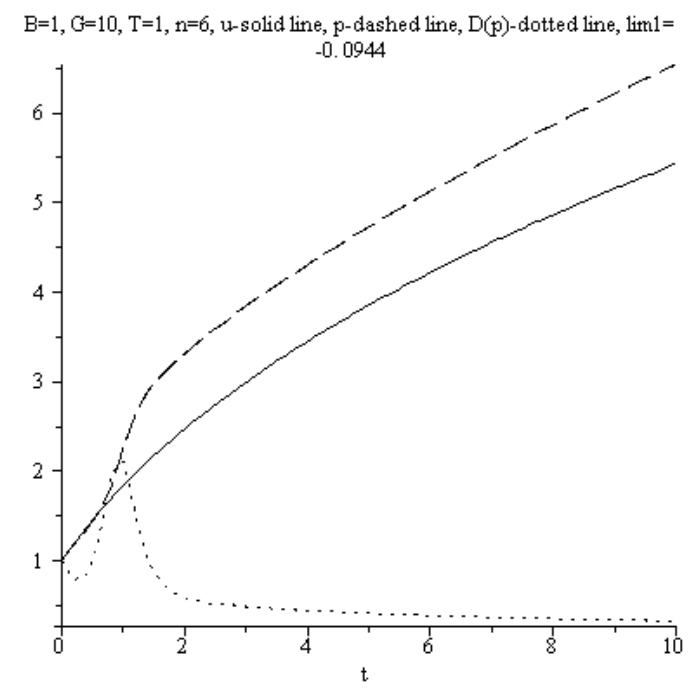

Figure 4.4. Evolution of $\tilde{u}(\tilde{x}), \tilde{p}(\tilde{x}), \partial \tilde{p}(\tilde{x}) / \partial \tilde{x} ; \tilde{B}=1, \tilde{\tau}=1, \tilde{g}_{x}=10, n=6$

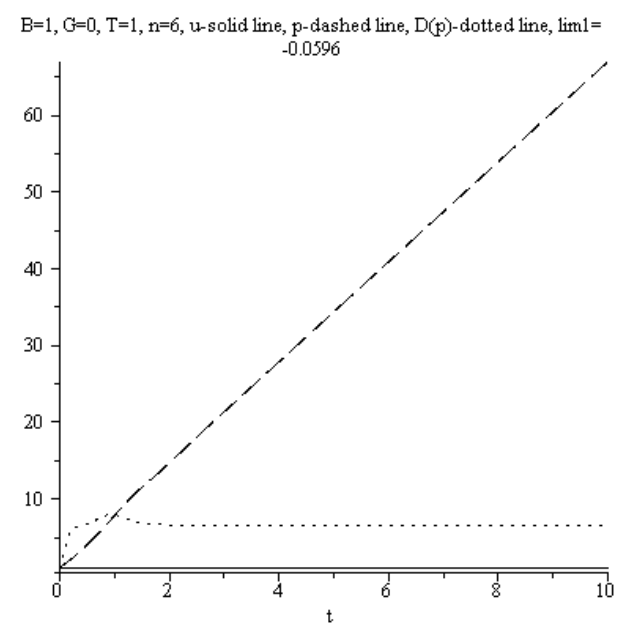

Figure 4.5. Evolution of $\tilde{u}(\tilde{x}), \tilde{p}(\tilde{x}), \partial \tilde{p}(\tilde{x}) / \partial \tilde{x} ; \tilde{B}=1, \tilde{\tau}=1, \tilde{g}_{x}=0, n=6$

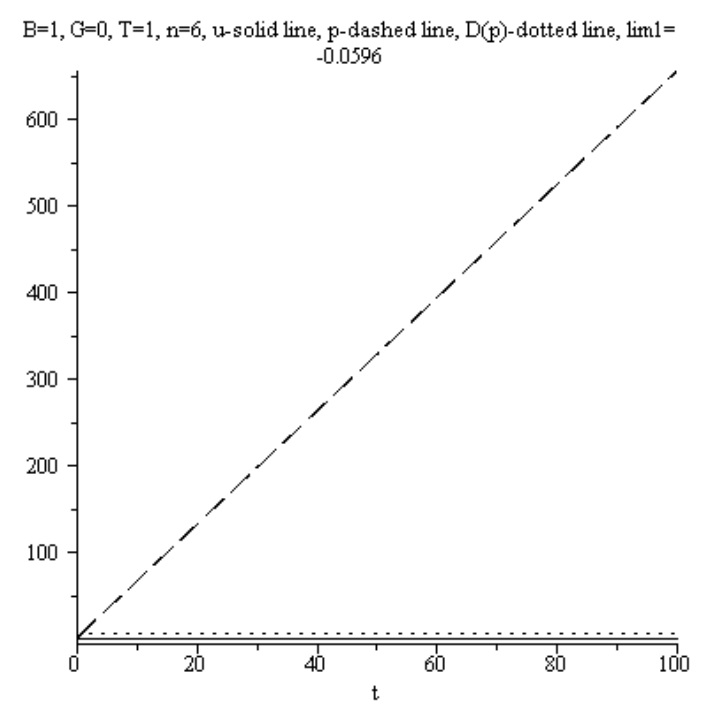

Figure 4.6. Evolution of $\tilde{u}(\tilde{x}), \tilde{p}(\tilde{x}), \partial \tilde{p}(\tilde{x}) / \partial \tilde{x} ; \tilde{B}=1, \tilde{\tau}=1, \tilde{g}_{x}=0, n=6$ 
The following figures 4.7, 4.8 show the result of modeling for different constant $\tilde{A}$ with the Cauchy conditions: $p(0)=1, u(0)=1, D(p)(0)=-1, \quad D(u)(0)=-1$.

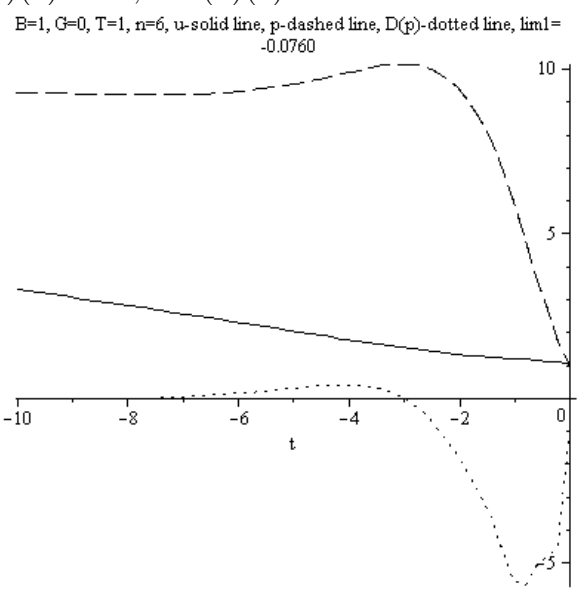

Figure 4.7. Evolution of $\tilde{u}(\tilde{x}), \tilde{p}(\tilde{x}), \partial \tilde{p}(\tilde{x}) / \partial \tilde{x} ; \tilde{B}=1, \tilde{\tau}=1, \tilde{g}_{x}=0, n=6$

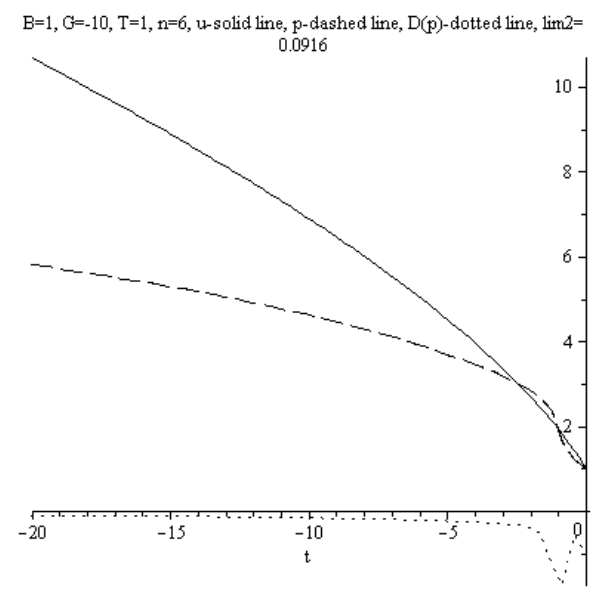

Figure 4.8. Evolution of $\tilde{u}(\tilde{x}), \tilde{p}(\tilde{x}), \partial \tilde{p}(\tilde{x}) / \partial \tilde{x} ; \tilde{B}=1, \tilde{\tau}=1, \tilde{g}_{x}=-10, n=6$.

Compare now the curves reflecting the influence of the external gravitational field on the PV evolution; for example the curves shown in the figures 3.8 and $3.10 ; 3.15$ and 3.16; 4.7 and 4.8. As we see the general features of the $\tilde{u}(\tilde{x}), \tilde{p}(\tilde{x}), \partial \tilde{p} / \partial \tilde{x}$ evolution demonstrate the different style of behavior.

\section{PV Evolution in the External Radial Gravitational Field. The Universe Evolution}

From the observations follow that about 13.7 billion years ago all the matter in the Universe was concentrated into a single incredibly small object. This object began to enlarge rapidly in a hot explosion, and it is still expanding today. Evidence for the Big Bang includes:

1. All the galaxies are moving away from us.

2. The further away a galaxy is, the faster it is moving away.

3. A cosmic microwave background radiation or CMBR was detected. This is received from all parts of the Universe and is thought to be the heat left over from the original explosion.

Let us investigate the situation after the explosion of a spherical object from the position of nonlocal physics. Then we consider a spherical PV object which is placed in the external radial gravitational field. The following conditions are fulfilled:

1. The physical picture corresponds to the $1 \mathrm{D}$ stationary situation. 
2. Nonlocal parameter $\tau$ is constant.

3. The external gravitational field is an arbitrary function of radius. It means that we have two kinds of forces - the self-consistent force $F_{r}$ (acting on the unit of volume) and known dependence $g_{r}(r)$ (external gravitational acceleration). Other unknown values - radial hydrodynamic velocity $v_{0 r}$ and PV pressure $p$ connected with the force $F_{r}$.

The nonlocal 1D hydrodynamic equations (2.1), (2.3), (2.5) have the form of continuity equation (non-stationary spherically symmetric case)

$$
\frac{\partial}{\partial t}\left\{\rho-\tau\left[\frac{\partial \rho}{\partial t}+\frac{1}{r^{2}} \frac{\partial\left(r^{2} \rho v_{0 r}\right)}{\partial r}\right]\right\}+\frac{1}{r^{2}} \frac{\partial}{\partial r}\left\{r^{2}\left\{\rho v_{0 r}-\tau\left[\frac{\partial}{\partial t}\left(\rho v_{0 r}\right)+\frac{1}{r^{2}} \frac{\partial\left(r^{2} \rho v_{0 r}^{2}\right)}{\partial r}-F_{r}\right]\right\}\right\}-\frac{1}{r^{2}} \frac{\partial}{\partial r}\left(\tau r^{2} \frac{\partial p}{\partial r}\right)=0
$$

where $\tau$ is a nonlocality parameter. The transfer to PV means the limit case $\rho \rightarrow 0$; as a result we have from (5.1)

$$
\frac{\partial}{\partial r}\left\{r^{2}\left[\frac{\partial p}{\partial r}-F_{r}\right]\right\}=0
$$

Equation (5.2) immediately can be integrated

$$
r^{2}\left[\frac{\partial p}{\partial r}-F_{r}\right]=C
$$

Obviously $\boldsymbol{C}=0$ and we obtain physically transparent equation

$$
F_{r}=\frac{\partial p}{\partial r}
$$

Momentum equation in the non-stationary spherically symmetric case is

$$
\begin{aligned}
& \frac{\partial}{\partial t}\left\{\rho v_{0 r}-\tau\left[\frac{\partial}{\partial t}\left(\rho v_{0 r}\right)+\frac{1}{r^{2}} \frac{\partial\left(r^{2} \rho v_{0 r}^{2}\right)}{\partial r}+\frac{\partial p}{\partial r}-F_{r}\right]\right\}-\left[F_{r}-\tau g_{r}\left(\frac{\partial \rho}{\partial t}+\frac{1}{r^{2}} \frac{\partial\left(r^{2} \rho v_{0 r}\right)}{\partial r}\right)\right] \\
& +\frac{1}{r^{2}} \frac{\partial}{\partial r}\left\{r^{2}\left\{\rho v_{0 r}^{2}-\tau\left[\frac{\partial}{\partial t}\left(\rho v_{0 r}^{2}\right)+\frac{1}{r^{2}} \frac{\partial\left(r^{2} \rho v_{0 r}^{3}\right)}{\partial r}-2 F_{r} v_{0 r}\right]\right\}\right\} \\
& +\frac{\partial p}{\partial r}-\frac{\partial}{\partial r}\left(\tau \frac{\partial p}{\partial t}\right)-2 \frac{\partial}{\partial r}\left(\frac{\tau}{r^{2}} \frac{\partial\left(r^{2} p v_{0 r}\right)}{\partial r}\right)-\frac{1}{r^{2}} \frac{\partial}{\partial r}\left(\tau r^{2} \frac{\partial\left(p v_{0 r}\right)}{\partial r}\right)=0
\end{aligned}
$$

The transfer to the stationary case in the PV theory means the limit case $\rho \rightarrow 0$, the result is Momentum equation in the stationary spherically symmetric case is

$$
\frac{2}{r^{2}} \frac{\partial}{\partial r}\left\{r^{2} F_{r} v_{0 r}\right\}-\frac{1}{r^{2}} \frac{\partial}{\partial r}\left\{r^{2} \frac{\partial\left(p v_{0 r}\right)}{\partial r}\right\}-2 \frac{\partial}{\partial r}\left(\frac{\tau}{r^{2}} \frac{\partial\left(r^{2} p v_{0 r}\right)}{\partial r}\right)=0
$$

or

$$
\frac{1}{r^{2}} \frac{\partial}{\partial r}\left\{r^{2} p \frac{\partial v_{0 r}}{\partial r}\right\}+2 \frac{\partial}{\partial r}\left(p \frac{\partial v_{0 r}}{\partial r}\right)+4 \frac{\partial}{\partial r}\left(\frac{1}{r} p v_{0 r}\right)+\frac{\partial}{\partial r}\left(v_{0 r} \frac{\partial p}{\partial r}\right)-\frac{2}{r} v_{0 r} \frac{\partial p}{\partial r}=0
$$

or

$$
\frac{\partial}{\partial r}\left[3 p \frac{\partial v_{0 r}}{\partial r}+v_{0 r} \frac{\partial p}{\partial r}+\frac{4}{r} p v_{0 r}\right]-\frac{2}{r}\left[v_{0 r} \frac{\partial p}{\partial r}-p \frac{\partial v_{0 r}}{\partial r}\right]=0
$$

Consider now the energy equation 


$$
\begin{aligned}
& \frac{\partial}{\partial t}\left\{\frac{1}{2} \rho v_{0 r}^{2}+\frac{3}{2} p-\tau\left[\frac{\partial}{\partial t}\left(\frac{1}{2} \rho v_{0 r}^{2}+\frac{3}{2} p\right)+\frac{1}{r^{2}} \frac{\partial}{\partial r}\left(r^{2} v_{0 r}\left(\frac{1}{2} \rho v_{0 r}^{2}+\frac{5}{2} p\right)\right)-F_{r} v_{0 r}\right]\right\} \\
& +\frac{1}{r^{2}} \frac{\partial}{\partial r}\left\{r ^ { 2 } \left\{\left(\frac{1}{2} \rho v_{0 r}^{2}+\frac{5}{2} p\right) v_{0 r}-\tau\left[\frac{\partial}{\partial t}\left(\left(\frac{1}{2} \rho v_{0 r}^{2}+\frac{5}{2} p\right) v_{0 r}\right)+\frac{1}{r^{2}} \frac{\partial}{\partial r}\left(r^{2}\left(\frac{1}{2} \rho v_{0 r}^{2}+\frac{7}{2} p\right) v_{0 r}^{2}\right)\right.\right.\right. \\
& \left.\left.\left.-F_{r} v_{0 r}^{2}-\left(\frac{1}{2} \rho v_{0 r}^{2}+\frac{3}{2} p\right) g_{r}\right]\right\}\right\}-\left\{F_{r} v_{0 r}-\tau\left[g_{r}\left(\frac{\partial}{\partial t}\left(\rho v_{o r}\right)+\frac{1}{r^{2}} \frac{\partial}{\partial r}\left(r^{2} \rho v_{0 r}^{2}\right)+\frac{\partial p}{\partial r}-F_{r}\right)\right]\right\} \\
& -\frac{1}{r^{2}} \frac{\partial}{\partial r}\left(\tau r^{2} \frac{\partial}{\partial r}\left(\frac{1}{2} p v_{0 r}^{2}+\frac{5}{2} \frac{p^{2}}{\rho}\right)\right)+\frac{1}{r^{2}} \frac{\partial}{\partial r}\left(r^{2} \tau p g_{r}\right)=0
\end{aligned}
$$

The transfer to the limit case $\rho \rightarrow 0$ leads to equation (stationary case)

$$
\begin{aligned}
& \frac{5}{r^{2}} \frac{\partial}{\partial r}\left[r^{2} p v_{0 r}\right]-\frac{1}{r^{2}} \frac{\partial}{\partial r}\left\{r^{2} \tau\left[\frac{7}{r^{2}} \frac{\partial}{\partial r}\left(r^{2} p v_{0 r}^{2}\right)-3 F_{r} v_{0 r}^{2}-3 p g_{r}\right]\right\} \\
& -2\left\{F_{r} v_{0 r}-\tau g_{r} \frac{1}{r^{2}} \frac{\partial}{\partial r}\left(r^{2} \rho v_{0 r}^{2}\right)\right\}-\frac{1}{r^{2}} \frac{\partial}{\partial r}\left(\tau r^{2} \frac{\partial}{\partial r}\left(p v_{0 r}^{2}+5 \frac{p^{2}}{\rho}\right)\right)+\frac{2}{r^{2}} \frac{\partial}{\partial r}\left(r^{2} \tau p g_{r}\right)=0
\end{aligned}
$$

or

$$
\begin{aligned}
& 5 p \frac{\partial v_{0 r}}{\partial r}+3 v_{0 r} \frac{\partial p}{\partial r}+\frac{10}{r} p v_{0 r}- \\
& -\frac{\partial}{\partial r}\left\{\tau\left[8 p \frac{\partial v_{0 r}^{2}}{\partial r}+5 v_{0 r}^{2} \frac{\partial p}{\partial r}+\frac{14}{r} p v_{0 r}^{2}-5 p g_{r}\right]\right\}- \\
& -\frac{2}{r} \tau\left[8 p \frac{\partial v_{0 r}^{2}}{\partial r}+5 v_{0 r}^{2} \frac{\partial p}{\partial r}+\frac{14}{r} p v_{0 r}^{2}-5 p g_{r}\right]+ \\
& +2 \tau F_{r} \frac{1}{r^{2}} \frac{\partial}{\partial r}\left(r^{2} v_{0 r}^{2}\right)-\frac{5}{r^{2}} \frac{\partial}{\partial r}\left(\tau r^{2} \frac{\partial}{\partial r}\left(\frac{p^{2}}{\rho}\right)\right)=0
\end{aligned}
$$

or

and finally

$$
\begin{aligned}
& 5 p \frac{\partial v_{0 r}}{\partial r}+3 v_{0 r} \frac{\partial p}{\partial r}+\frac{10}{r} p v_{0 r}- \\
& -\tau \frac{\partial}{\partial r}\left[5 v_{0 r}^{2} \frac{\partial p}{\partial r}+16 p v_{0 r} \frac{\partial v_{0 r}}{\partial r}-5 p g_{r}\right]-60 \tau p \frac{1}{r} v_{0 r} \frac{\partial v_{0 r}}{\partial r}-10 \tau \frac{1}{r} v_{0 r}^{2} \frac{\partial p}{\partial r}- \\
& -\frac{10}{r} \tau v_{0 r}^{2} \frac{\partial p}{\partial r}+\frac{10}{r} \tau p g_{r}+2 \tau F_{r} \frac{\partial v_{0 r}^{2}}{\partial r}-14 \tau p v_{0 r}^{2} \frac{1}{r^{2}}-\frac{5}{r^{2}} \frac{\partial}{\partial r}\left(\tau r^{2} \frac{\partial}{\partial r}\left(\frac{p^{2}}{\rho}\right)\right)=0
\end{aligned}
$$

$$
\begin{aligned}
& 5 p \frac{\partial v_{0 r}}{\partial r}+3 v_{0 r} \frac{\partial p}{\partial r}+\frac{10}{r} p v_{0 r}- \\
& -\tau \frac{\partial}{\partial r}\left[5 v_{0 r}^{2} \frac{\partial p}{\partial r}+16 p v_{0 r} \frac{\partial v_{0 r}}{\partial r}-5 p g_{r}\right]-20 \tau \frac{1}{r} v_{0 r}^{2} \frac{\partial p}{\partial r}-60 \tau p \frac{1}{r} v_{0 r} \frac{\partial v_{0 r}}{\partial r}+ \\
& +\frac{10}{r} \tau p g_{r}-14 \tau p v_{0 r}^{2} \frac{1}{r^{2}}+4 \tau v_{0 r} \frac{\partial p}{\partial r} \frac{\partial v_{0 r}}{\partial r}-\frac{5}{r^{2}} \frac{\partial}{\partial r}\left(\tau r^{2} \frac{\partial}{\partial r}\left(\frac{p^{2}}{\rho}\right)\right)=0
\end{aligned}
$$

The last term of the energy equation (5.13) correspond as before to the external energy fluctuation and will be omitted. In other words we intend to consider the PV evolution under influence of the perturbations of the Cauchy conditions. Therefore we reach the following system of equation (SYSTEM 3) written in the dimensionless form using the scales 


$$
\begin{gathered}
{\left[r_{0}\right],\left[u_{0}\right],\left[p_{0}\right],[F]=\frac{p_{0}}{r_{0}},\left[t_{0}\right]=\frac{r_{0}}{u_{0}}, r_{0}=u_{0} t_{0},\left[g_{r}\right]=\frac{u_{0}^{2}}{r_{0}} .} \\
\tilde{F}_{r}=\frac{\partial \tilde{p}}{\partial \tilde{r}} \\
\frac{\partial}{\partial \tilde{r}}\left[3 \tilde{p} \frac{\partial \tilde{v}_{0 r}}{\partial \tilde{r}}+\tilde{v}_{0 r} \frac{\partial \tilde{p}}{\partial \tilde{r}}+\frac{4}{\tilde{r}} \tilde{p} \tilde{v}_{0 r}\right]-\frac{2}{\tilde{r}}\left[\tilde{v}_{0 r} \frac{\partial \tilde{p}}{\partial \tilde{r}}-\tilde{p} \frac{\partial \tilde{v}_{0 r}}{\partial \tilde{r}}\right]=0 \\
5 \tilde{p} \frac{\partial \tilde{v}_{0 r}}{\partial \tilde{r}}+3 \tilde{v}_{0 r} \frac{\partial \tilde{p}}{\partial \tilde{r}}+\frac{10}{\tilde{r}} \tilde{p} \tilde{v}_{0 r}- \\
-\tilde{\tau} \frac{\partial}{\partial \tilde{r}}\left\{5 \tilde{v}_{0 r}^{2} \frac{\partial \tilde{p}}{\partial \tilde{r}}+16 \tilde{p} \tilde{v}_{0 r} \frac{\partial \tilde{v}_{0 r}}{\partial \tilde{r}}-5 \tilde{p} \tilde{g}_{r}\right\}-\frac{20}{\tilde{r}} \tilde{\tau} \tilde{v}_{0 r}^{2} \frac{\partial \tilde{p}}{\partial \tilde{r}}-\frac{60}{\tilde{r}} \tilde{\tau} \tilde{p} \tilde{v}_{0 r} \frac{\partial \tilde{v}_{0 r}}{\partial \tilde{r}}+ \\
+\frac{10}{\tilde{r}} \tilde{\tau} \tilde{p} \tilde{g}_{r}-\frac{14}{\tilde{r}^{2}} \tilde{\tau} \tilde{p} \tilde{v}_{0 r}^{2}+4 \tilde{\tau} \tilde{v}_{0 r} \frac{\partial \tilde{p}}{\partial \tilde{r}} \frac{\partial \tilde{v}_{0 r}}{\partial \tilde{r}}=0 .
\end{gathered}
$$

Let us introduce the approximation

$$
\tilde{g}_{r}=\frac{\mathrm{G}}{\tilde{r}^{2}}
$$

In this case SYSTEM 4 contains two dimensionless parameters $\mathrm{G}, \mathrm{T} \leftrightarrow \tilde{\tau}$ and needs four Cauchy conditions. These conditions we write down for the external surface of the spherical object. Then we investigate the evolution of the surface perturbation on the following scenario of the PV behavior.

In the theory of Big Bang the external gravitational field is absent. Let on the surface of a spherical PV object appears the perturbation of physical parameters in the absence of the external gravitation $(\mathrm{G}=0)$, namely

$$
p(1)=1, u(1) \leftrightarrow v_{0 r}(1)=1, \quad D(p)(1)=1, \quad D(u)(1)=1
$$

Figures (5.1) - (5.14) demonstrate the results of calculations for changing $\tau$. The information required is indicated in the figures and in figure captions including the boundaries of the solution existence.

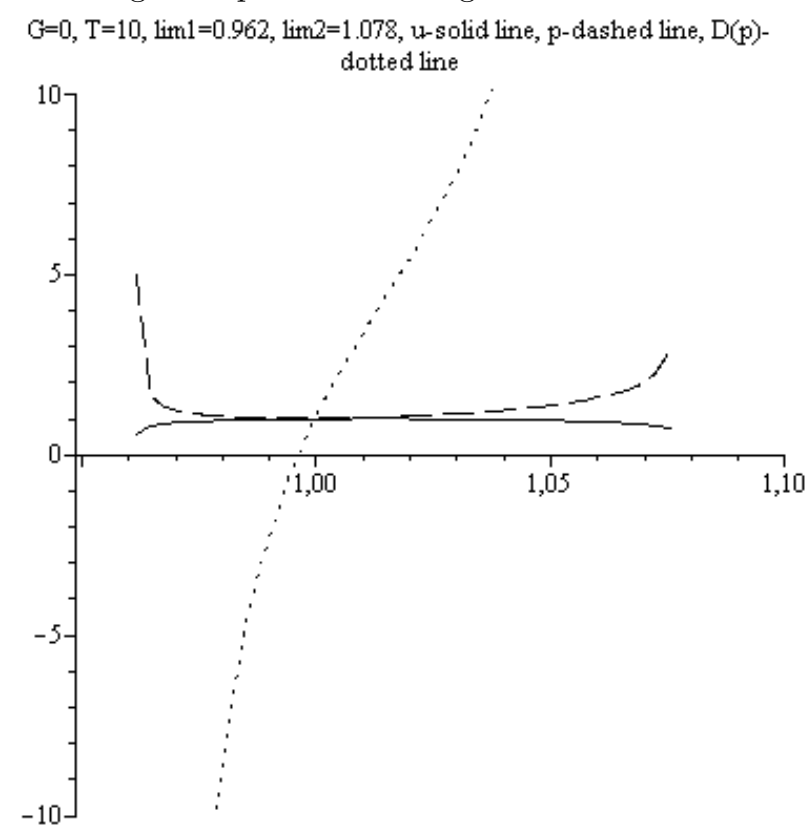

Figure 5.1. Evolution of $\tilde{u}(\tilde{r}), \tilde{p}(\tilde{r}), \partial \tilde{p}(\tilde{r}) / \partial \tilde{r}=F(\tilde{r}) ; \tilde{\tau}=10, \mathrm{G}=0$ 


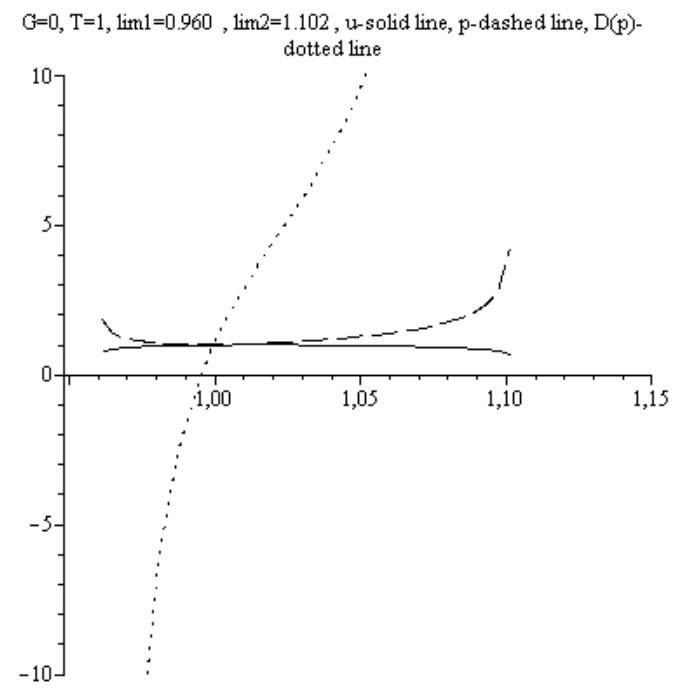

Figure 5.2. Evolution of $\tilde{u}(\tilde{r}), \tilde{p}(\tilde{r}), \partial \tilde{p}(\tilde{r}) / \partial \tilde{r} ; \tilde{\tau}=1, \mathrm{G}=0$

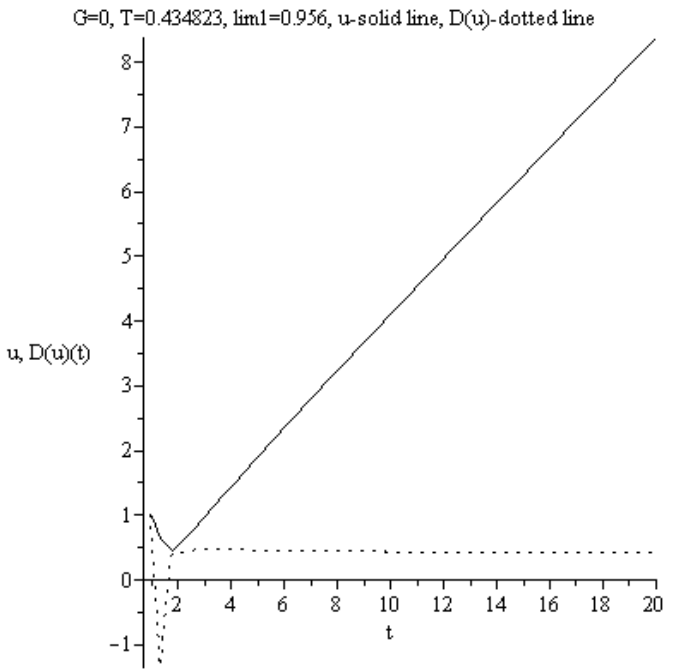

Figure 5.3. Evolution of $\tilde{u}(\tilde{r}), \partial \tilde{u}(\tilde{r}) / \partial \tilde{r} ; \tilde{\tau}=0.434823, \mathrm{G}=0$

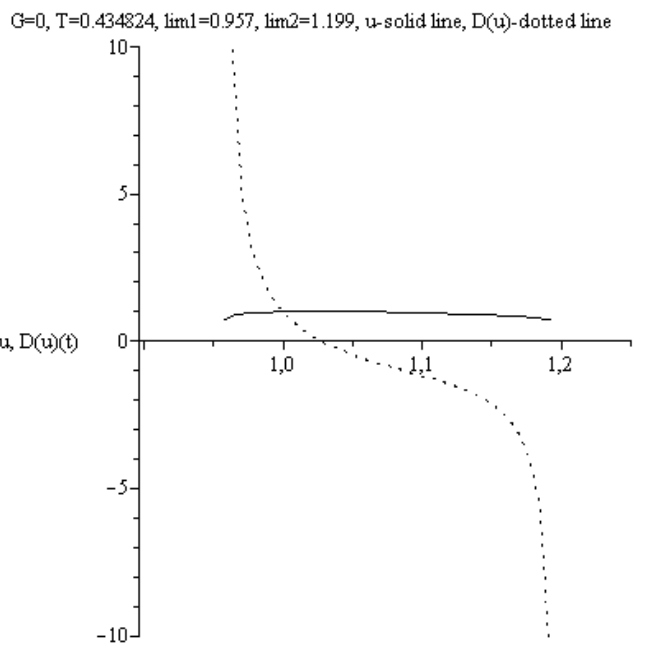

Figure 5.4. Evolution of $\tilde{u}(\tilde{r}), \partial \tilde{u}(\tilde{r}) / \partial \tilde{r} ; \tilde{\tau}=0.434824, \mathrm{G}=0$. 


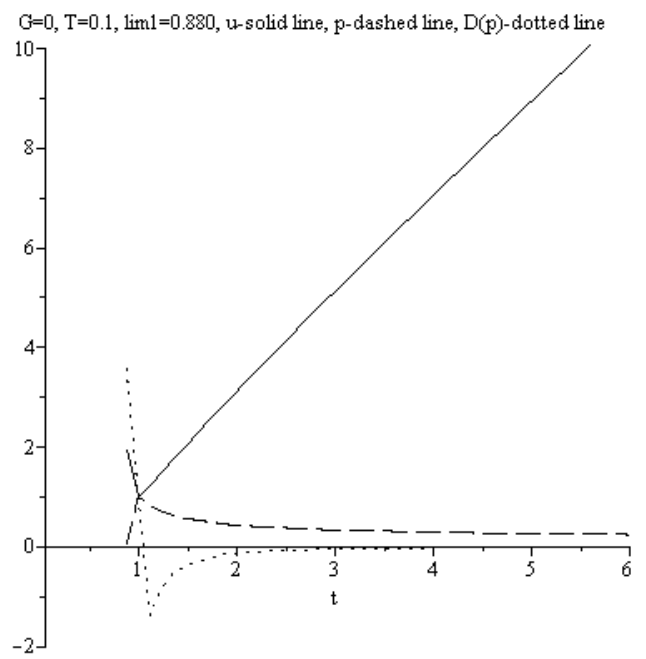

Figure 5.5. Evolution of $\tilde{u}(\tilde{r}), \tilde{p}(\tilde{r}), \partial \tilde{p}(\tilde{r}) / \partial \tilde{r} ; \tilde{\tau}=0.1, \mathrm{G}=0$

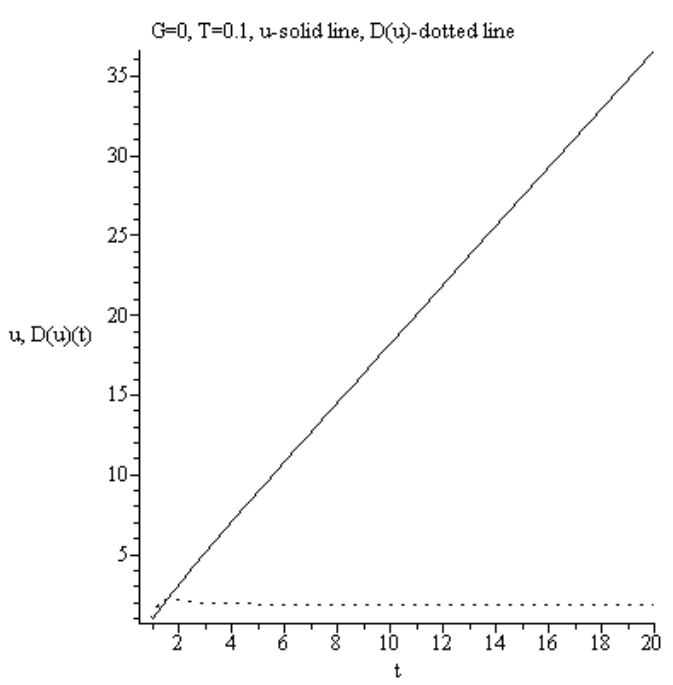

Figure 5.6. Evolution of $\tilde{u}(\tilde{r}), \partial \tilde{u}(\tilde{r}) / \partial \tilde{r} ; \tilde{\tau}=0.1, \mathrm{G}=0$.

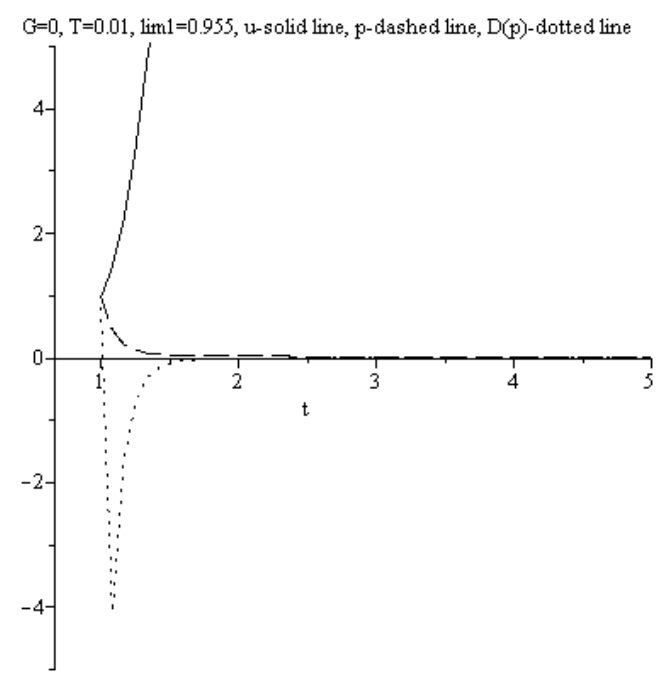

Figure 5.7. Evolution of $\tilde{u}(\tilde{r}), \tilde{p}(\tilde{r}), F(\tilde{r}) ; \tilde{\tau}=0.01, \mathrm{G}=0$ 


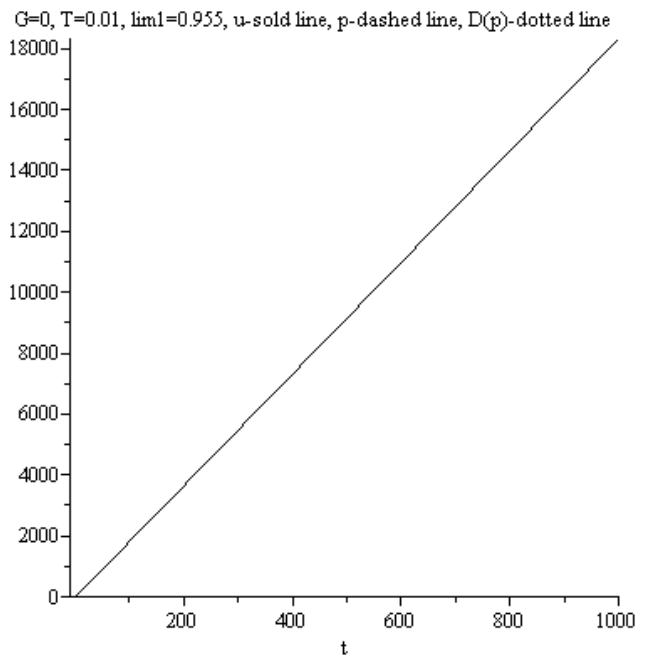

Figure 5.8. Evolution of $\tilde{u}(\tilde{r}) ; \tilde{\tau}=0.01, \mathrm{G}=0$

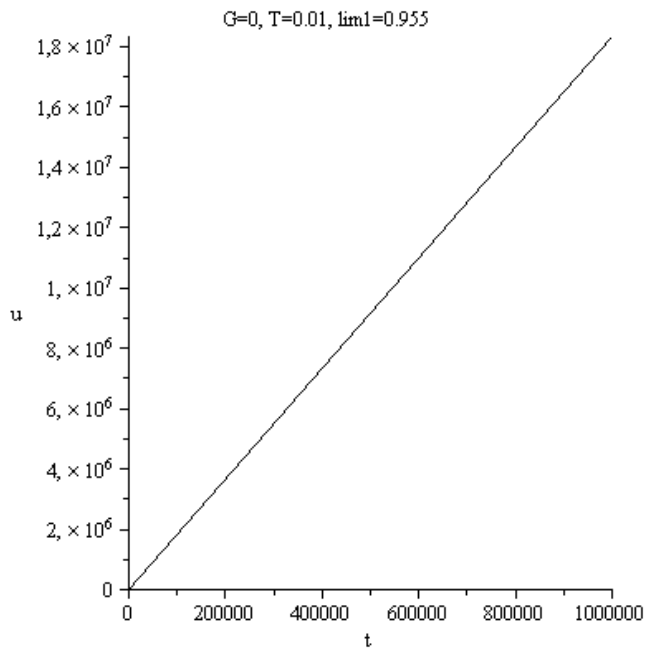

Figure 5.9. Evolution of $\tilde{u}(\tilde{r}) ; \tilde{\tau}=0.01, \mathrm{G}=0$

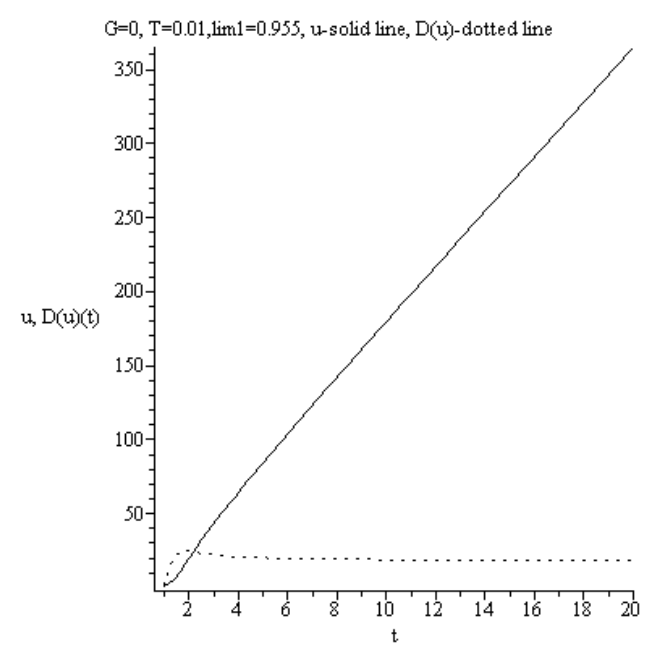

Figure 5.10. Evolution of $\partial \tilde{u}(\tilde{r}) / \partial \tilde{r} ; \tilde{\tau}=0.01, G=0$ 


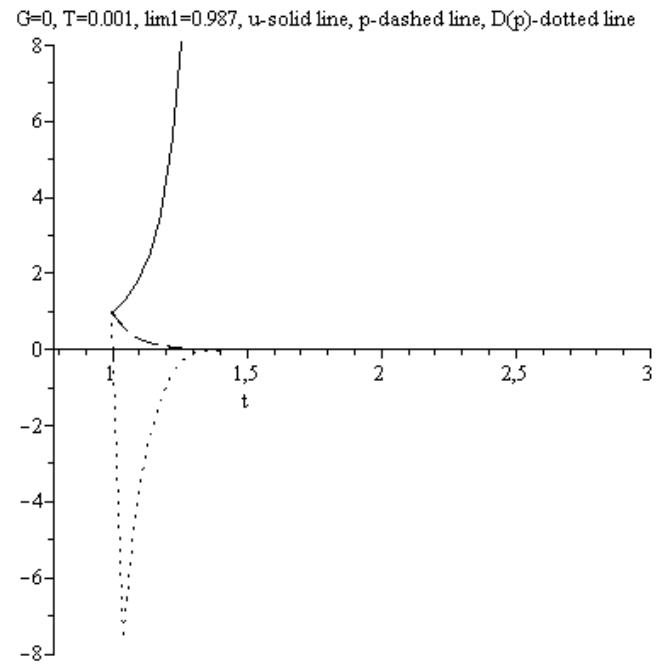

Figure 5.11. Evolution of $\tilde{u}(\tilde{r}), \tilde{p}(\tilde{r}), \partial \tilde{p}(\tilde{r}) / \partial \tilde{r} ; \tilde{\tau}=0.001, \mathrm{G}=0$.

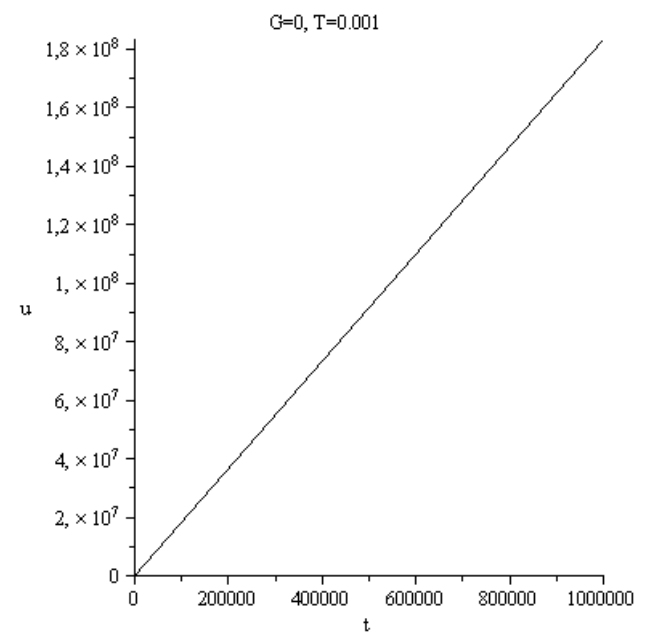

Figure 5.12. Evolution of $\tilde{u}(\tilde{r}) ; \tilde{\tau}=0.001, \mathrm{G}=0$.

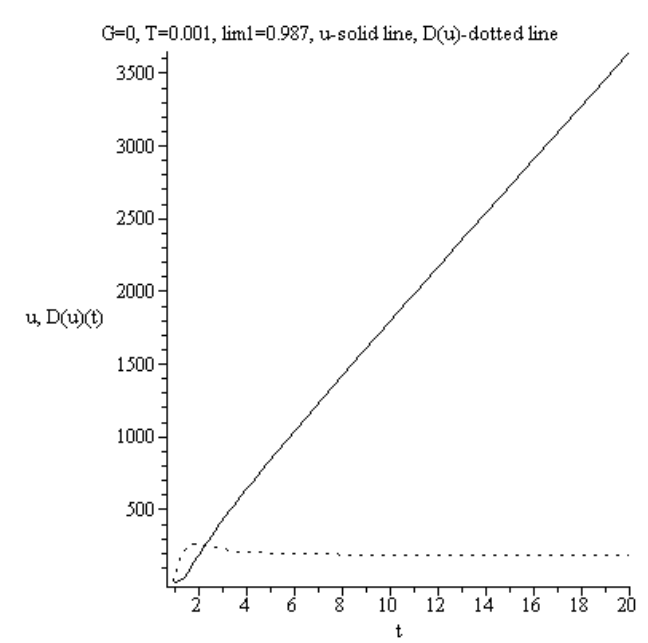

Figure 5.13. Evolution of $\tilde{u}(\tilde{r}), \partial \tilde{u}(\tilde{r}) / \partial \tilde{r} ; \tilde{\tau}=0.001, \mathrm{G}=0$ 


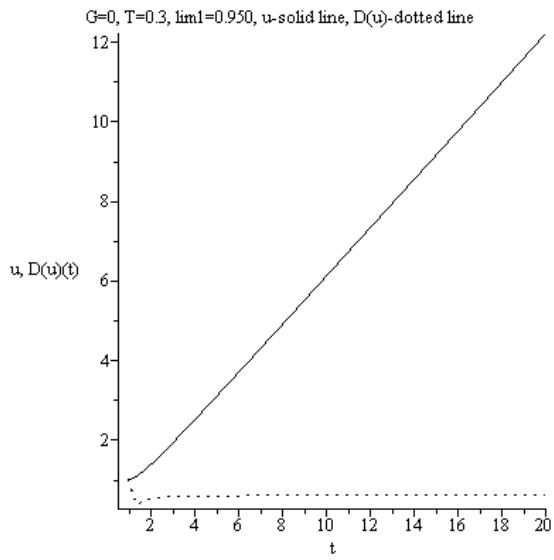

Figure 5.14. Evolution of $\tilde{u}(\tilde{r}), \partial \tilde{u}(\tilde{r}) / \partial \tilde{r} ; \tilde{\tau}=0.3, \mathrm{G}=0$

\section{Conclusion}

1. The Universe evolution leads to the finite size of this system if the nonlocality parameter $\tilde{\tau} \sim 1$ (figures 5.1 - 5.3).

2. Nonlocal coefficient $\tilde{\tau}$ plays the role of fundamental constant in the Universe. The critical $\tilde{\tau}_{c r}$ exists when the infinite Universe transforms into the finite Universe (see figures 5.3 and 5.4). For this model $\tilde{\tau}_{c r}=0.4348235$.

3. If $\tilde{\tau} \approx 0.1$ or less the Universe exists in the generalized Hubble regime (figures 5.6-5.14).

4. If $\tilde{\tau}$ is not too small the generalized Hubble regime contains the "regime with acceleration" when the Hubble coefficient $H(\tilde{r})$ is not constant and increasing with the distance $\tilde{r}$. But with the $\tilde{r}$ increasing the regime with acceleration transforms into classical Hubble regime with the constant $H, \tilde{u}(\tilde{r})=\tilde{H} \tilde{r}$. This result should be verified by observations.

5. Varying $\tilde{\tau}$ leads to the principally different the Universe evolution before achieving the Hubble regime. Look at figures 5.6, 5.10, 5.13 and 5.14. In the cases reflected in figures 5.6, 5.10, 5.13 we have our Universe with accelerations in the first stage of evolution. But the situation reflected in the figure 5.14 corresponds to the Universe with deceleration of the Universe expanding in the first stage of its evolution.

6. Diminishing of the nonlocal parameter $\tilde{\tau}$ leads to the Hadamard effect - the smaller the parameter $\tilde{\tau}$ the greater the rate of expansion (compare figures 5.6, 5.9, 5.10 and 5.12).

Let us estimate $\tilde{\tau}$ for our Universe. The Hubble expression can be written in the dimensionless form

$$
\frac{\tilde{u}}{\tilde{r}}=\tilde{H}
$$

where the velocity scale $u_{0}^{i}$ is the velocity of the initial radial perturbation and $r_{0}^{i}$ is the radius of the initial object. It means that the dimensionless Hubble parameter can be written as

$$
\tilde{H}=H \frac{r_{0}^{i}}{v_{0}^{i}}
$$

The ratio $r_{0}^{i} / u_{0}^{i}$ corresponds to the early times of the Universe evolution. For our time $r_{0}^{n} / u_{0}^{n}$ can be estimated as the age of the Universe and within the $\Lambda \mathrm{CDM}$ model is about $(4,354 \pm 0,012) \cdot 10^{17} \mathrm{~s}$. In this case

$$
\frac{\tilde{u}}{\tilde{r}}=\tilde{H}=H \frac{r_{0}^{n}}{u_{0}^{n}} \cong 2.169 \cdot 10^{-18} \cdot 4.35 \cdot 10^{17}=0.943
$$

Other estimates of the age of the Universe lead to much greater values of $\tilde{H}$ (and therefore to smaller values of the parameter $\tilde{\tau}$ ). Nevertheless we can formulate some conclusions using figures 5.5 - 5.7, 5.9, 5.10, 5.12, 5.13:

1. We are living in the young Universe with varying $\tilde{H}(\tilde{r})$. 
2. The origin of the Universe can not be considered as singular point. In other words it means that initial size of our Universe is not much less that the character size of the visible now Universe.

3. If the initial radius of the object tends to infinity, the physical picture is changing radically. This situation is investigated in the next Item.

Let us consider now the PV evolution placed in the external gravitational field. The following figures $5.15-5.17$ contain the analogues results but for $\mathrm{G}=1$.

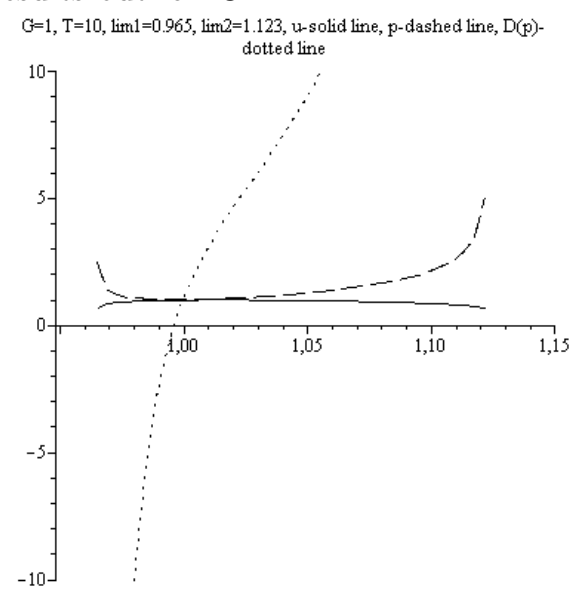

Figure 5.15. Evolution of $\tilde{u}(\tilde{r}), \tilde{p}(\tilde{r}), \partial \tilde{p}(\tilde{r}) / \partial \tilde{r} ; \tilde{\tau}=10, \mathrm{G}=1$

$\mathrm{G}=1, \mathrm{~T}=1, \lim 1=0.962$, lim2 $=1.275$, u-solid line, $\mathrm{p}$-dashed line, $\mathrm{D}(\mathrm{p})$-dotted

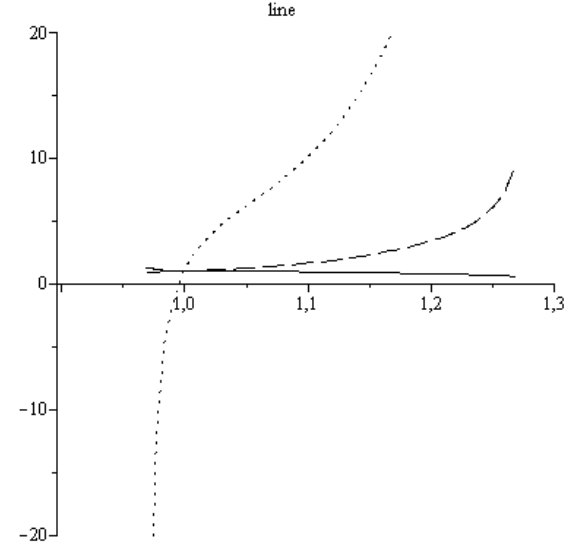

Figure 5.16. Evolution of $\tilde{u}(\tilde{r}), \tilde{p}(\tilde{r}), \partial \tilde{p}(\tilde{r}) / \partial \tilde{r} ; \tilde{\tau}=1, \mathrm{G}=1$

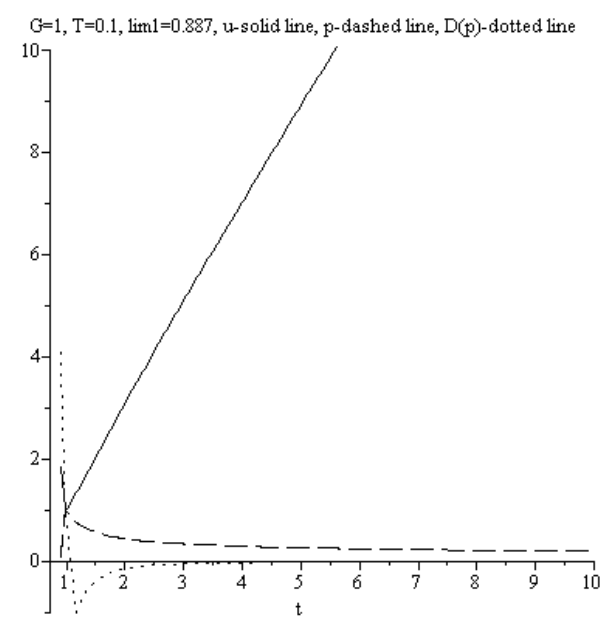

Figure 5.17. Evolution of $\tilde{u}(\tilde{r}), \tilde{p}(\tilde{r}), \partial \tilde{p}(\tilde{r}) / \partial \tilde{r} ; \tilde{\tau}=0.1, \mathrm{G}=1$ 
As we see from figures 5.15 - 5.17 diminishing of parameter $\tau$ leads to the significant reconstruction of the integral curves - typical effect of the theory differential equations with the small parameter in front of the senior derivatives. The other important effect - in many cases (see for example figures 5.5, 5.7) the perturbation on the surface leads to the PV-object reconstruction only in a narrow domain near the mentioned surface.

Figures 5.18 and 5.19 correspond to strong gravitational fields $(\mathrm{G}=1000)$.

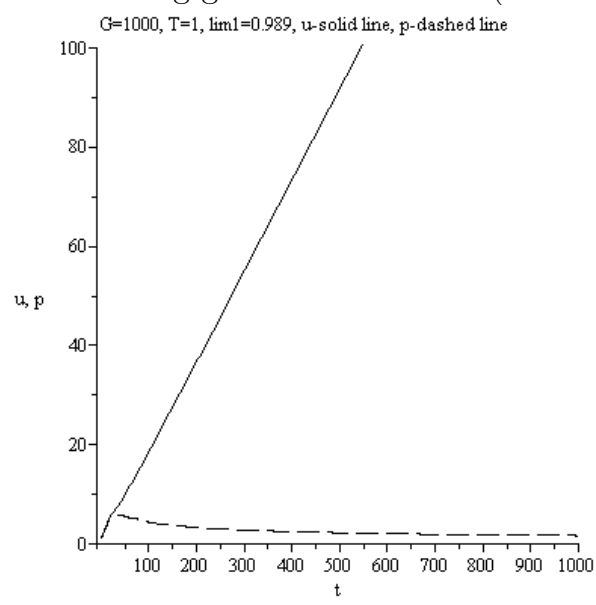

Figure. 5.18. Evolution of $\tilde{u}(\tilde{r}), \tilde{p}(\tilde{r}) ; \tilde{\tau}=1, \mathrm{G}=1000$

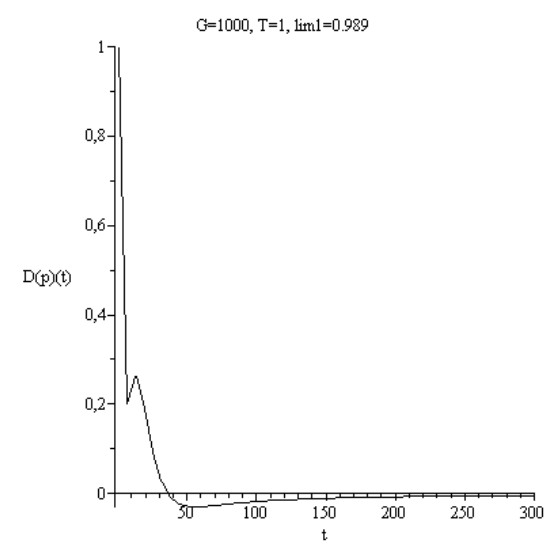

Figure 5.19. Evolution of $\tilde{F}(\tilde{r})=\partial \tilde{p}(\tilde{r}) / \partial \tilde{r} ; \tilde{\tau}=1, \mathrm{G}=1000$

The figures 5.20 and 5.21 reflect the result of modeling for the gravitational field of forces opposite direction.

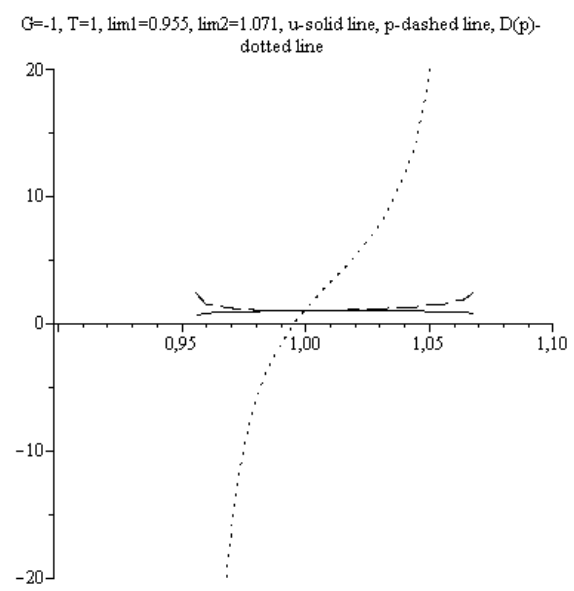

Figure 5.20. Evolution of $\tilde{u}(\tilde{r}), \tilde{p}(\tilde{r}), \partial \tilde{p}(\tilde{r}) / \partial \tilde{r} ; \tilde{\tau}=1, \mathrm{G}=-1$ 


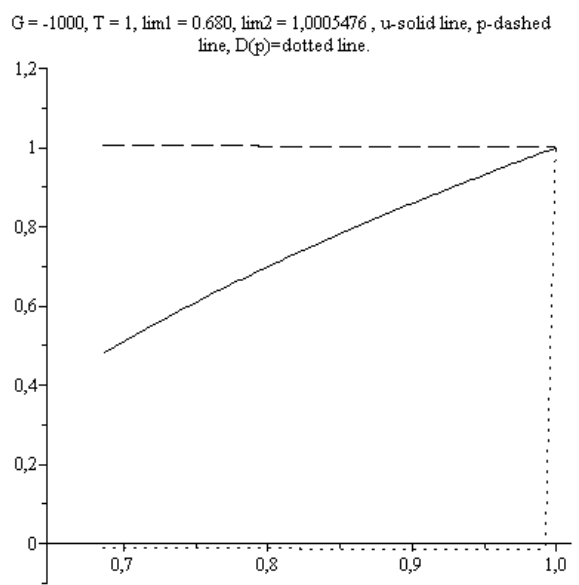

Figure 5.21. Evolution of $\tilde{u}(\tilde{r}), \tilde{p}(\tilde{r}), \partial \tilde{p}(\tilde{r}) / \partial \tilde{r} ; \tilde{\tau}=1, \mathrm{G}=-1000$

The last figures $5.22-5.24$ show the influence of the negative gradients, namely

$$
p(1)=1, u(1) \leftrightarrow v_{0 r}(1)=1, \quad D(p)(1)=-1, \quad D(u)(1)=-1
$$

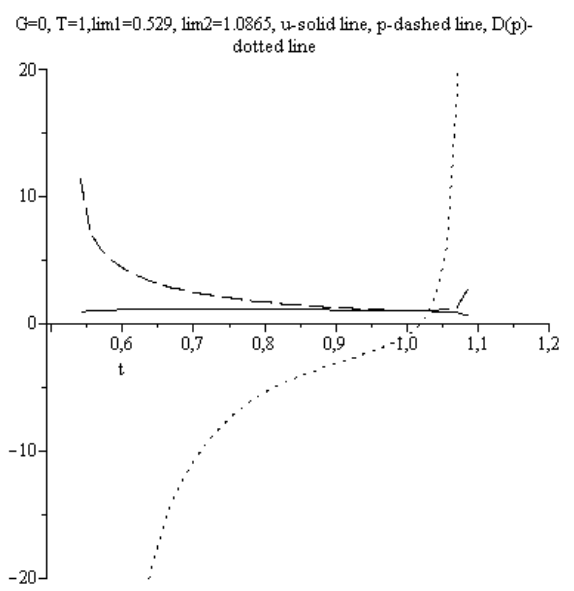

Figure 5.22. Evolution of $\tilde{u}(\tilde{r}), \tilde{p}(\tilde{r}), \partial \tilde{p}(\tilde{r}) / \partial \tilde{r} ; \tilde{\tau}=1, \mathrm{G}=0$.

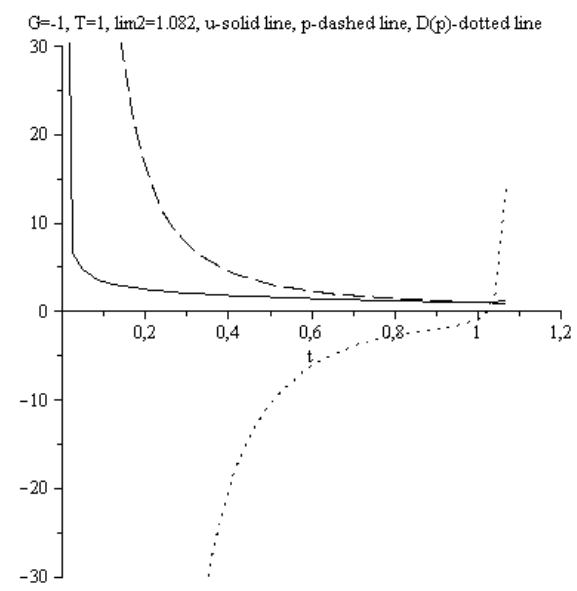

Figure 5.23. Evolution of $\tilde{u}(\tilde{r}), \tilde{p}(\tilde{r}), \partial \tilde{p}(\tilde{r}) / \partial \tilde{r} ; \tilde{\tau}=1, \mathrm{G}=-1$. 


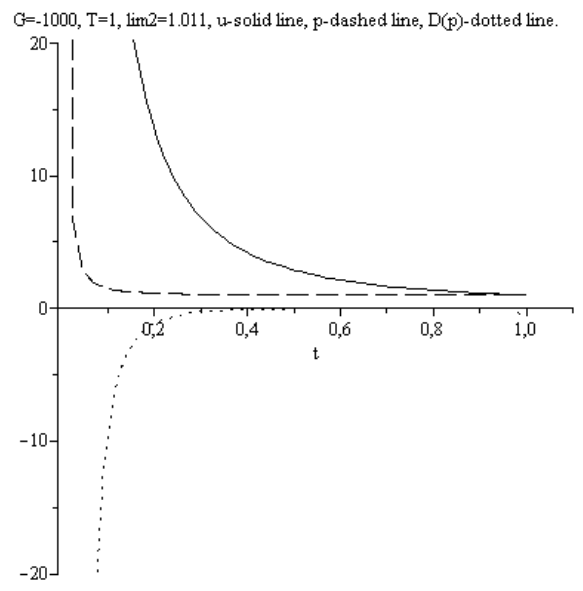

Figure 5.24. Evolution of $\tilde{u}(\tilde{r}), \tilde{p}(\tilde{r}), \partial \tilde{p}(\tilde{r}) / \partial \tilde{r} ; \tilde{\tau}=1, \mathrm{G}=-1000$.

\section{Semi-Analytic Solutions in the Nonlocal Theory with Gravitation}

Let us write down the system of equations (5.14)-(5.17) for the limit case of the large radius. We find

$$
\begin{gathered}
\frac{\partial}{\partial \tilde{r}}\left[3 \tilde{p} \frac{\partial \tilde{v}_{0 r}}{\partial \tilde{r}}+\tilde{v}_{0 r} \frac{\partial \tilde{p}}{\partial \tilde{r}}\right]=0 \\
5 \tilde{p} \frac{\partial \tilde{v}_{0 r}}{\partial \tilde{r}}+3 \tilde{v}_{0 r} \frac{\partial \tilde{p}}{\partial \tilde{r}}-\tilde{\tau} \frac{\partial}{\partial \tilde{r}}\left\{5 \tilde{v}_{0 r}^{2} \frac{\partial \tilde{p}}{\partial \tilde{r}}+16 \tilde{p} \tilde{v}_{0 r} \frac{\partial \tilde{v}_{0 r}}{\partial \tilde{r}}-5 \tilde{p} \tilde{g}_{r}\right\}+4 \tilde{\tau} \tilde{v}_{0 r} \frac{\partial \tilde{p}}{\partial \tilde{r}} \frac{\partial \tilde{v}_{0 r}}{\partial \tilde{r}}=0 .
\end{gathered}
$$

After integration of (6.2) we find

$$
3 \tilde{p} \frac{\partial \tilde{v}_{0 r}}{\partial \tilde{r}}+\tilde{v}_{0 r} \frac{\partial \tilde{p}}{\partial \tilde{r}}=0
$$

or

$$
\tilde{v}_{0 r}^{3} \tilde{p}=C
$$

Using (6.4) we have from (6.3)

$$
2 \frac{\partial}{\partial \tilde{r}}\left(\tilde{p} \tilde{v}_{0 r}\right)-\tilde{\tau} \frac{\partial}{\partial \tilde{r}}\left\{\tilde{p} \tilde{v}_{0 r} \frac{\partial \tilde{v}_{0 r}}{\partial \tilde{r}}-5 \tilde{p} \tilde{g}_{r}\right\}+4 \tilde{\tau} \tilde{v}_{0 r} \frac{\partial \tilde{p}}{\partial \tilde{r}} \frac{\partial \tilde{v}_{0 r}}{\partial \tilde{r}}=0 .
$$

The application of the analytic solution (6.5) obtains

$$
2 \frac{\partial}{\partial \tilde{r}} \frac{1}{\tilde{v}_{0 r}^{2}}-\tilde{\tau} \frac{\partial}{\partial \tilde{r}}\left\{\frac{1}{\tilde{v}_{0 r}^{2}} \frac{\partial \tilde{v}_{0 r}}{\partial \tilde{r}}-5 \frac{1}{\tilde{v}_{0 r}^{3}} \tilde{g}_{r}\right\}+4 \tilde{\tau} \tilde{v}_{0 r} \frac{\partial \tilde{v}_{0 r}}{\partial \tilde{r}} \frac{\partial}{\partial \tilde{r}} \frac{1}{\tilde{v}_{0 r}^{3}}=0
$$

or

$$
\tilde{\tau} \tilde{v}_{0 r}^{2} \frac{\partial^{2} \tilde{v}_{0 r}}{\partial \tilde{r}^{2}}+\left(4 \tilde{v}_{0 r}+15 \tilde{\tau} \tilde{g}_{r}+10 \tilde{\tau} \tilde{v}_{0 r}\left(\frac{\partial \tilde{v}_{0 r}}{\partial \tilde{r}}\right)\right) \frac{\partial \tilde{v}_{0 r}}{\partial \tilde{r}}-5 \tilde{\tau} \tilde{v}_{0 r} \frac{\partial \tilde{g}_{r}}{\partial \tilde{r}}=0 .
$$

Let us use the dependence $\tilde{g}(\tilde{r})$ in the form

$$
\tilde{g}(\tilde{r})=\frac{G}{\tilde{r}^{2}}
$$

In this case

$$
\tilde{\tau} \tilde{v}_{0 r}^{2} \frac{\partial^{2} \tilde{v}_{0 r}}{\partial \tilde{r}^{2}}+\left(4 \tilde{v}_{0 r}+10 \tilde{\tau} \tilde{v}_{0 r}\left(\frac{\partial \tilde{v}_{0 r}}{\partial \tilde{r}}\right)-15 \tilde{\tau} \frac{G}{\tilde{r}^{2}}\right) \frac{\partial \tilde{v}_{0 r}}{\partial \tilde{r}}-10 \tilde{\tau} \tilde{v}_{0 r} \frac{G}{\tilde{r}^{3}}=0
$$


The ordinary differential equation (6.10) can be easily integrated by numerical way. Let us show some corresponding results using for example Cauchy conditions

$$
\tilde{v}_{0 r}(1)=1, \frac{\partial \tilde{v}_{0 r}}{\partial \tilde{r}}(1)=1
$$

Figures 6.1-6.11 reflect the result of numerical calculations as the Maple application.

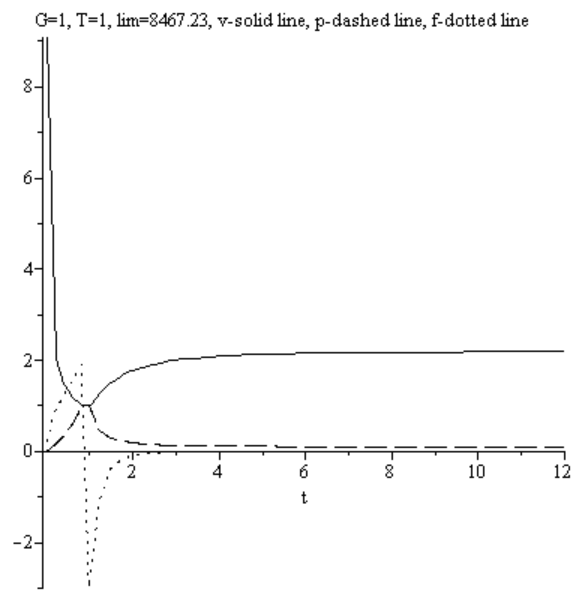

Figure 6.1. Dependence $\tilde{v}(\tilde{r}), \tilde{p}(\tilde{r}), \tilde{F}(\tilde{r}), G=1, \quad \tilde{\tau}=1$

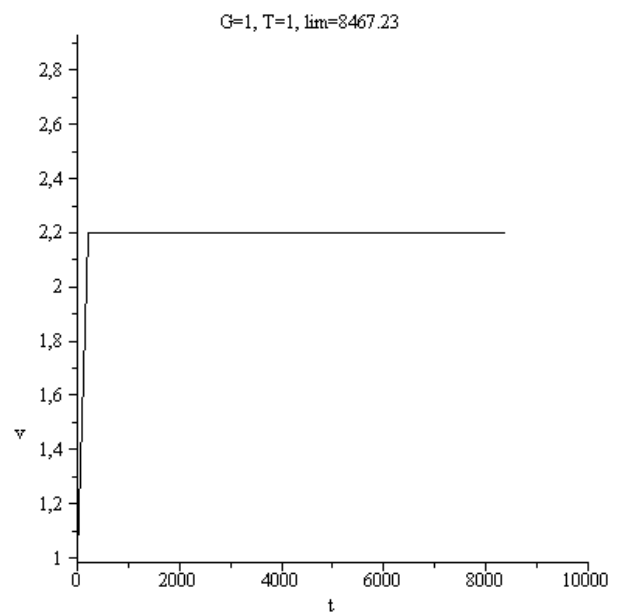

Figure 6.2. Dependence $\tilde{v}(\tilde{r}), G=1, \quad \tilde{\tau}=1$

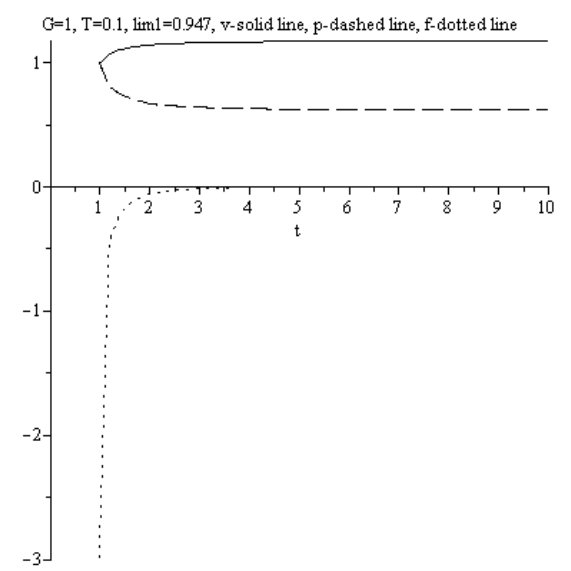

Figure 6.3. Dependence $\tilde{v}(\tilde{r}), \tilde{p}(\tilde{r}), \tilde{F}(\tilde{r}), G=1, \quad \tilde{\tau}=0.1$ 


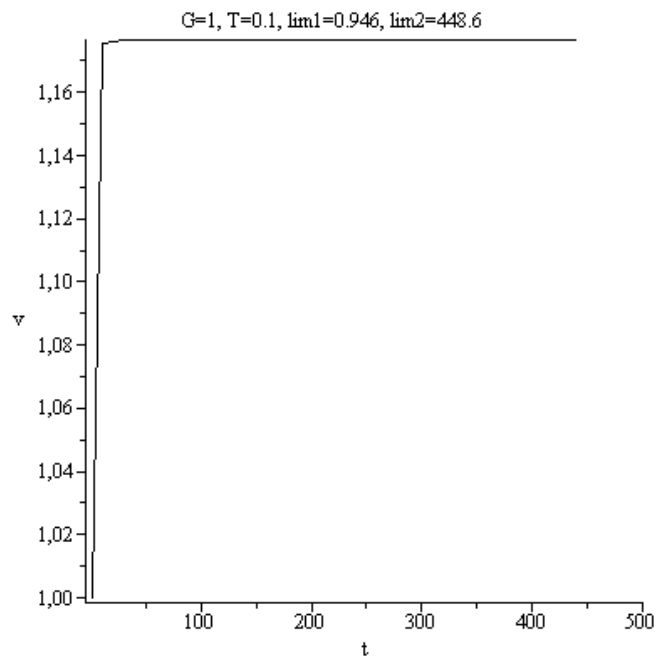

Figure 6.4. Dependence $\tilde{v}(\tilde{r}), G=1, \quad \tilde{\tau}=0.1$

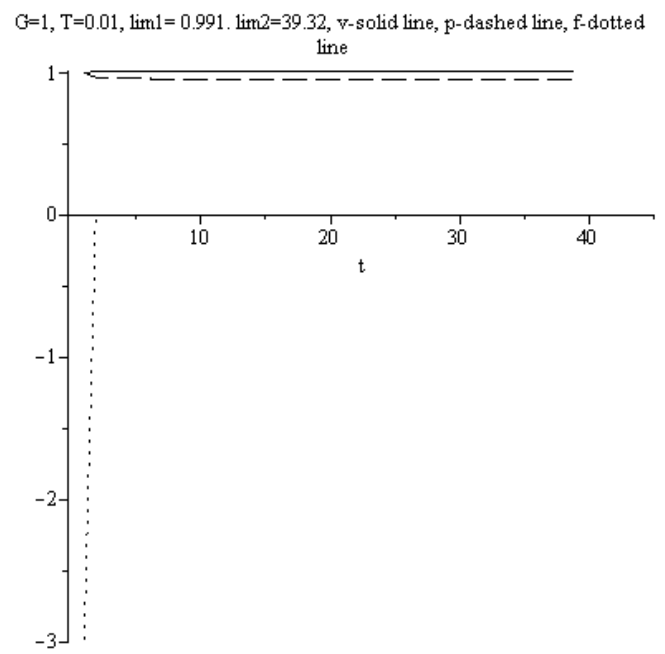

Figure 6.5. Dependence $\tilde{v}(\tilde{r}), \tilde{p}(\tilde{r}), \tilde{F}(\tilde{r}), G=1, \quad \tilde{\tau}=0.1$

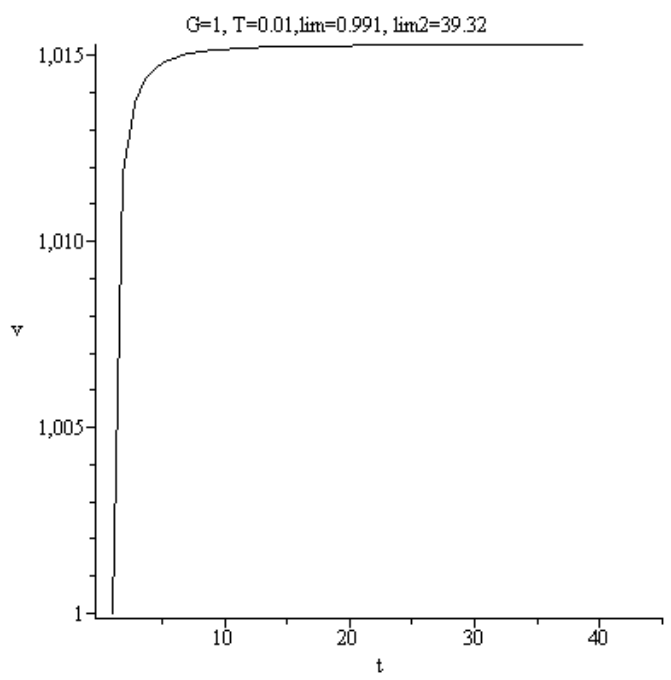

Figure 6.6. Dependence $\tilde{v}(\tilde{r}), G=1, \tilde{\tau}=0.01$ 


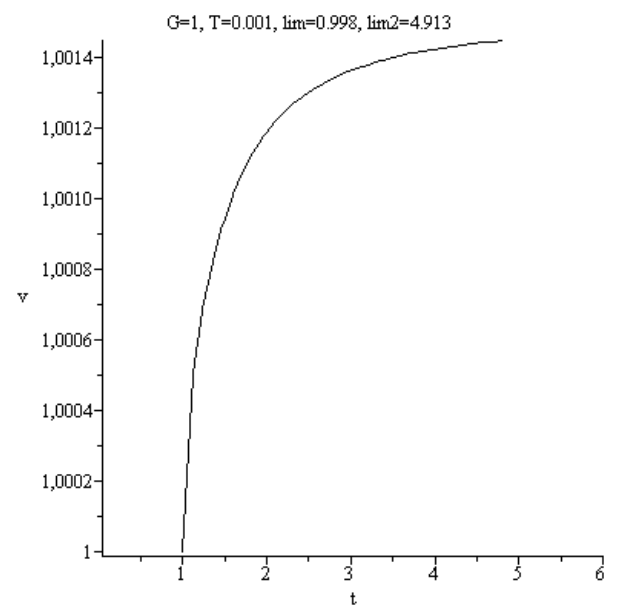

Figure 6.7. Dependence $\tilde{v}(\tilde{r}), G=1, \quad \tilde{\tau}=0.001$

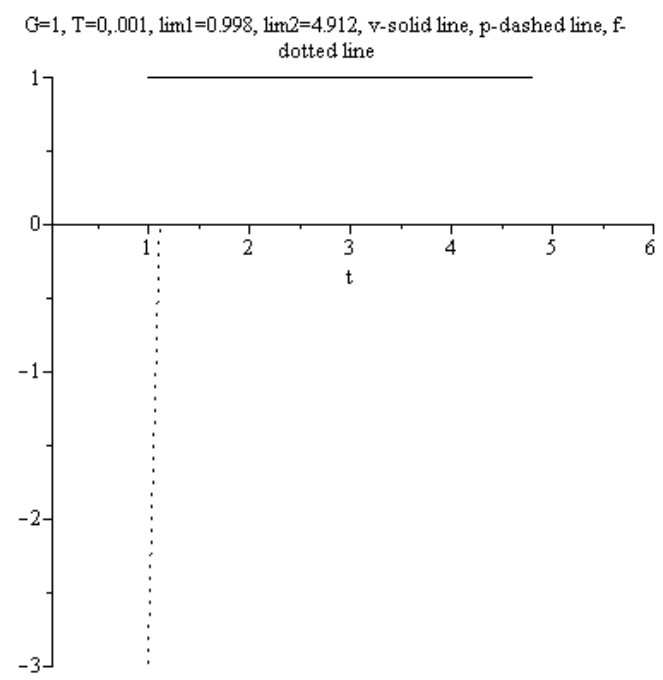

Figure 6.8. Dependence $\tilde{v}(\tilde{r}), \tilde{p}(\tilde{r}), \tilde{F}(\tilde{r}), G=1, \tilde{\tau}=0.001$

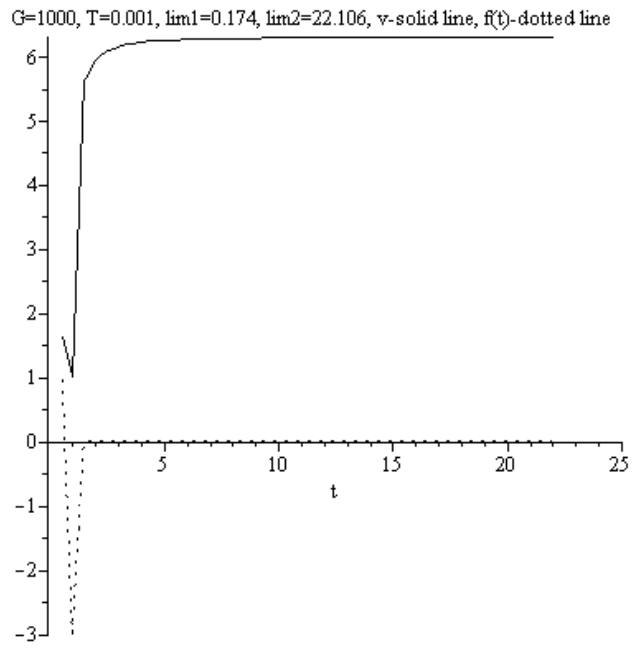

Figure 6.9. Dependence $\tilde{v}(\tilde{r}), \tilde{F}(\tilde{r}), G=1000, \tilde{\tau}=0.001$ 


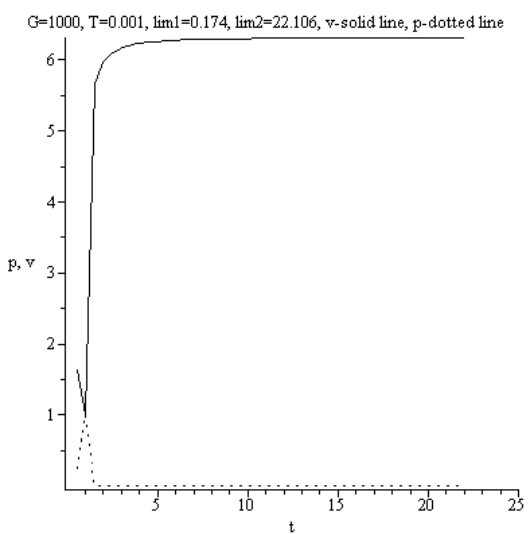

Figure 6.10. Dependence $\tilde{v}(\tilde{r}), \tilde{p}(\tilde{r}), G=1000, \tilde{\tau}=0.001$

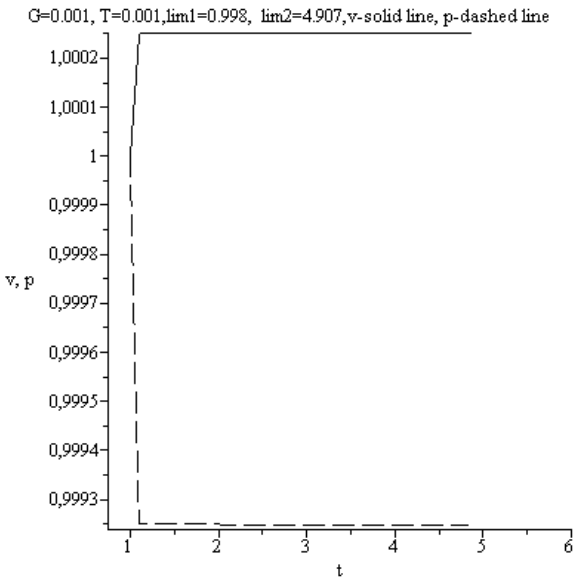

Figure 6.11. Dependence $\tilde{v}(\tilde{r}), \tilde{p}(\tilde{r}), G=1000, \tilde{\tau}=0.001$

If the external gravitational field does not exist we have from $(6.10)$

$$
\tilde{\tau} \tilde{v}_{0 r}^{2} \frac{\partial^{2} \tilde{v}_{0 r}}{\partial \tilde{r}^{2}}+\left(4 \tilde{v}_{0 r}+10 \tilde{\tau} \tilde{v}_{0 r}\left(\frac{\partial \tilde{v}_{0 r}}{\partial \tilde{r}}\right)\right) \frac{\partial \tilde{v}_{0 r}}{\partial \tilde{r}}=0 .
$$

Apart of the particular solution $\tilde{v}_{0 r}=$ const this equation has the general analytical solution via $\mathrm{W}$ Lambert function which leads to unique solution of the equation in the considered conditions. But it is simpler to use the numerical methods. The following figures 6.12 and 6.13 reflect the calculations for different $\tilde{\tau}$.

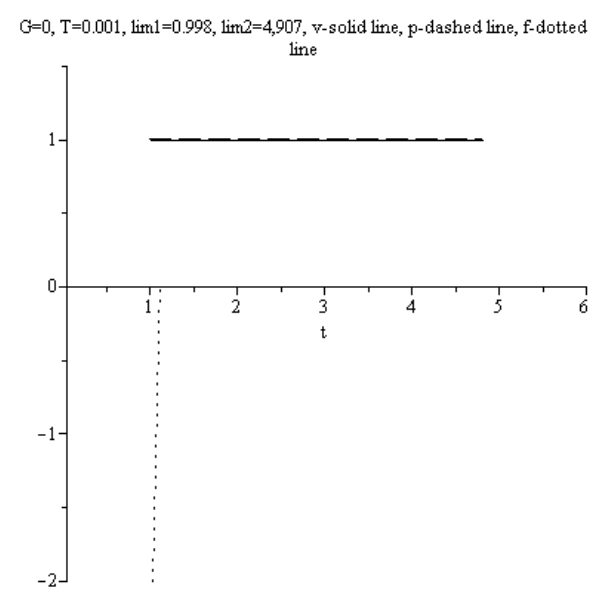

Figure 6.12. Dependence $\tilde{v}(\tilde{r}), \tilde{p}(\tilde{r}), \tilde{F}(\tilde{r}), G=0, \quad \tilde{\tau}=0.001$. 


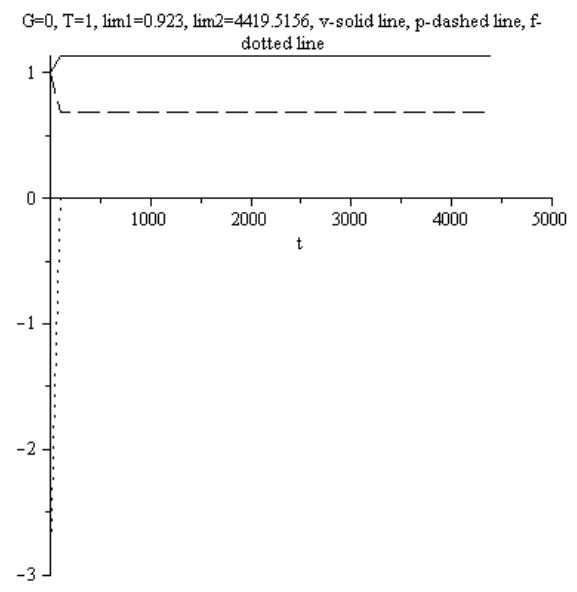

Figure 6.13. Dependence $\tilde{v}(\tilde{r}), \tilde{p}(\tilde{r}), \tilde{F}(\tilde{r}), G=0, \tilde{\tau}=1$

\section{Conclusion}

1. Known dependence $\tilde{v}(\tilde{r})$ realizes immediately the calculation $\tilde{p}(\tilde{r})$ using (6.5) and $\tilde{F}_{r}$ using (6.1).

2. All mentioned calculations lead to so to speak "volume quantization" - the solutions exist only in the finite domain of space.

3. The linear size of these domains is diminishing with the $\tilde{\tau}$ diminishing. The figure captions include the boundaries of the solution existence. It means that the nonlocal theory leads to the explosion of object whose size is significantly less than the size of the visible Universe.

\section{Some General Remarks and Conclusions}

Remind the basic conclusions following in particular from the results of mathematical modeling: 1. Nonlocal hydrodynamics contains not only quantum hydrodynamics [1-4] but also the limit cases for the density $\rho \rightarrow \infty$ (black hole) and $\rho \rightarrow 0$ (physical vacuum including Big Bang theory).

2. Processes in PV-engine can be considered for the case $\rho \neq 0$ as well. The initial Cauchy conditions provide the greatest influence on the results of the calculations.

3. The main result consists in the affirmation that the physical system "works" in regimes of attraction and repulsion. It is direct consequence of nonlocal physics.

4. Appearance of the initial gradients and the external gravitational field leads to moving of the PVengine as a whole system, which has no attitude to the usual jet propulsion. If the initial gradients and gravitational force have the opposite directions, the space PV evolution is closed in the finite space domain.

5. The calculations are realized in the vast diapason of parameter changing and Cauchy conditions. In considered cases we find the strong influence of the external gravitational and electromagnetic fields on PV evolution and therefore on the PV Engine. If this effect can be independently confirmed, it is certainly a revolution in physics and technology.

6. The PV evolution near the massive objects (like stars) strongly depends on the force of the external gravitation.

\section{References}

1. Alexeev B.V., Generalized Boltzmann Physical Kinetics. Elsevier Amsterdam, The Netherlands (2004) 368p.

2. Alexeev B.V., Unified Non-local Theory of Transport Processes, Elsevier Amsterdam, The Netherlands (2015) 644p.

3. Alexeev B.V., Unified Non-local Relativistic Theory of Transport Processes, Elsevier Amsterdam, The Netherlands (2016) 455p. 
4. Alexeev B.V., Nonlocal Astrophysics. Dark matter, Dark Energy, Physical Vacuum. Elsevier Amsterdam, The Netherlands (2017) 454p.

5. Alexeev B.V. Transport processes in physical vacuum. Advances in Astrophysics, 2017 (to be published).

6. Alexeev B.V. To the nonlocal theory of waves in physical vacuum. Advances in Astrophysics, 2017 (to be published).

7. Lebedew P., Untersuchungen über die Druckkräfte des Lichtes, «Annalen der Physik», 1901, fasc. 4, Bd 6, S. 433-458. DOI: https://dx.doi.org/10.1002/andp.19013111102;

8. Shawyer, Roger, "Second generation EmDrive propulsion applied to SSTO launcher and interstellar probe," Acta Astronautica. 116: 166-174. (1 November 2015). doi:10.1016/j.actaastro.2015.07.002.

9. Juan Yang, et al., Prediction and Experimental Measurement of the Electromagnetic Thrust Generated by Microwave Thruster System, NWPU Xi'an China, Chinese Physical Society and IOP Publishing Ltd., 2013.

10. Yang, J.; Liu, X.-C.; Wang, Y.-G.; Tang, M.-J.; Luo, L.-T.; Jin, Y.-Z.; Ning, Z.-X. (February 2016). "Thrust Measurement of an Independent Microwave Thruster Propulsion Device with Three-Wire Torsion Pendulum Thrust Measurement System". Journal of Propulsion Technology (in Chinese). 37 (2): 362-371.

11. D. Brady, et al., Anomalous thrust production from an RF test device measured on a low thrust torsion pendulum. NASA USA IAA Joint Propulsion Conference, Cleveland, 2014.

12. H. White, P. March, W. Nehemiah, W. O'Neill, Eagleworks Laboratories: Advanced Propulsion Physics Research. NASA Technical Reports Server (NTRS) (Technical report). NASA. JSC-CN-25207 (5 December 2011).

13. H. White, P. March, J. Lawrence, J Vera, A. Sylvester, D. Brady and P. Bailey, Thrust from a Closed RadioFrequency Cavity in Vacuum, NASA Johnson Space Center, Houston, Texas 77058 DOI: 10.2514/1.B36120, Journal of Propulsion and Power, Vol. 33, No. 4, July-August 2017, p. 830-841. 